\title{
Bias Evaluation and Reduction in 3D OP-OSEM Reconstruction in Dynamic Equilibrium PET Studies
}

\author{
Cláudia Régio Brambilla ( $\nabla$ c.regio-brambilla@fz-juelich.de ) \\ Forschungszentrum Julich GmbH https://orcid.org/0000-0002-3735-8511 \\ Jürgen Scheins
}

Forschungszentrum Julich $\mathrm{GmbH}$

Ahlam Issa

Forschungszentrum Julich $\mathrm{GmbH}$

\section{Lutz Tellmann}

Forschungszentrum Julich $\mathrm{GmbH}$

Hans Herzog

Forschungszentrum Julich $\mathrm{GmbH}$

\section{Elena Rota Kops}

Forschungszentrum Julich GmbH

N. Jon Shah

Forschungszentrum Julich $\mathrm{GmbH}$

Irene Neuner

Forschungszentrum Julich $\mathrm{GmbH}$

Christoph W. Lerche

Forschungszentrum Julich GmbH

Original research

Keywords: binding potential, dynamic PET, low statistics, neuroreceptor studies, quantitation bias

Posted Date: June 22nd, 2020

DOI: https://doi.org/10.21203/rs.3.rs-35926/v1

License: (c) (i) This work is licensed under a Creative Commons Attribution 4.0 International License.

Read Full License 
1 Bias Evaluation and Reduction in 3D OP-OSEM Reconstruction in Dynamic

\section{Equilibrium PET Studies}

Cláudia Régio Brambilla ${ }^{* 1,2}$, Jürgen Scheins ${ }^{1}$, Ahlam Issa ${ }^{1}$, Lutz Tellmann ${ }^{1}$, Hans Herzog ${ }^{1}$, Elena Rota Kops $^{1}$, N. Jon Shah ${ }^{1,3,4,5}$, Irene Neuner ${ }^{1,2,4}$, Christoph W. Lerche ${ }^{1}$

${ }^{1}$ Institute of Neuroscience and Medicine, INM-4, Forschungszentrum Jülich GmbH, Wilhelm-Johnen-Straße, 52428 Jülich, Germany

${ }^{2}$ Department of Psychiatry, Psychotherapy and Psychosomatics, RWTH Aachen University, Pauwelsstraße 30, 52074 Aachen, Germany

${ }^{3}$ Institute of Neuroscience and Medicine, INM-11, Forschungszentrum Jülich GmbH, Wilhelm-Johnen-Straße, 52428 Jülich, Germany

${ }^{4}$ JARA - BRAIN - Translational Medicine, RWTH Aachen University, Pauwelsstraße 30, 52074 Aachen, Germany ${ }^{5}$ Department of Neurology, RWTH Aachen University, Pauwelsstraße 30, 52074 Aachen, Germany

*Corresponding author: Cláudia R. Brambilla - c.regio-brambilla@fz-juelich.de Wilhelm-Johnen-Straße, 52428, INM-4 Forschungszentrum Jülich GmbH, Germany Building: 15.16, Room: 1023 Tel.: +49 2461 61-96856 Fax: +49 2461-61-2089

j.scheins@fz-juelich.de a.issa@fz-juelich.de 1.tellmann@fz-juelich.de hans.r.herzog@gmx.de e.rota.kops@fz-juelich.de n.j.shah@fz-juelich.de i.neuner@fz-juelich.de c.lerche@fz-juelich.de 
33 Abstract

34 Background: Iterative image reconstruction algorithms are widely used in positron emission 35 tomography (PET). However, they are known to contribute to quantitation bias, which is 36 particularly pronounced during dynamic PET studies such as neuroreceptor binding studies with

$37{ }^{11} \mathrm{C}$-labelled radiotracers where count rates become low towards the end of the examination. This 38 problem is relevant in case simultaneous PET/MR studies which apply a bolus-infusion protocol to allow the multimodal comparison between control or resting state and stimulation effects in a 40 single session of e.g. 60 min, i.e. 3 half-lives of ${ }^{11}$ C. A quantitation bias may interfere with 41 stimulation related changes. In this study, we evaluated the impact of the 3D ordinary Poisson 42 OSEM (3D OP-OSEM) on quantitation accuracy reconstructions and the subsequent propagation 43 into binding potential values using a decay study of a ${ }^{11} \mathrm{C}$ filled phantom and a human brain data set. To evaluate the reconstruction bias, we tested different reconstruction framing schemes and

45 propose a framing scheme that keeps the counts per time frame constant over the full acquisition 46 time. We also compared the vendor's 3D OP-OSEM image reconstruction method to an in-house 47 developed reconstruction (PRESTO toolkit).

48 Results: In general, a bias for low and high activity concentration regions was observed in the 49 range of $\pm 3 \%$ and $-3 \%$ to $5 \%$, respectively. Using the alternative proposed framing scheme, a 50 lower bias was achieved for regions with low activity concentration using PRESTO, and a 51 stabilization of the mean bias for the binding potential was achieved at around 5\% with the 52 vendor's reconstruction throughout the relevant activity curve time interval.

53 Conclusions: The bias in activity concentration propagated into the binding potential values 54 leading to a mean bias of around 5\%, thus allowing the detection of changes in binding values 55 during equilibrium of $>5 \%$. 
56 Keywords: binding potential, dynamic PET, low statistics, neuroreceptor studies, quantitation 57 bias.

58

59

60

61

62

63

64

65

66 67 and thus low counts of the radioligand per frame.

68

69

70

71

72

73

74

75

76

77 78

\section{Background}

Iterative image reconstruction algorithms based on the known maximum likelihood - expectation maximization (ML-EM) have been widely used in positron emission tomography (PET) for the past three decades, with the order-subset (OS) variants being particularly prevalent (1-4). However, these methods have been shown to cause bias for applications involving low count levels (5-9). This effect is often problematic in dynamic PET studies with ${ }^{11} \mathrm{C}$-labelled radioligands and especially in the case of neuroreceptor binding studies that usually use reference regions, e.g. cerebellum or pons/brainstem, which frequently present low neuroreceptor concentrations levels

Other groups using ML-EM based reconstruction methods have reported different levels of bias (7) and overestimation, as well as underestimation in volumes of interest (VOIs) with either low or high activity concentrations (10). In the case of 3D OSEM based reconstruction algorithms, the source of the bias has been attributed to the introduction of a positive bias in the reconstructed images due to the non-negativity constraint in the data prior to correction (11). To avoid bias from the non-negativity constraint, 3D ordinary Poisson OSEM (OP-OSEM) can be used as an alternative iterative reconstruction method as it implements all required data corrections in a way that preserves non-negativity during the reconstruction $(6,12)$. However, 3D OP-OSEM uses observed random and scattered coincidence events without updating them for each iteration step during the image reconstruction (13). The accuracy of this method has been studied and a bias of $10 \%$ or more was reported (11) in regions of a homogeneous phantom. Byars and colleagues (14) 
79 showed that the bias can be reduced when a variance reduction algorithm (VRR) is implemented 80 to reduce the variance in estimated random counts. However, it is important to consider that other

81 factors can also contribute to the bias in image reconstruction at low count rates, including scatter 82 correction implementations (e.g. frame-based) or the framing scheme $(10,15,16)$.

83 The aim of this study was to evaluate the impact of different framing schemes and reconstruction 84 procedures on the quantitative accuracy of PET images using a ${ }^{11} \mathrm{C}$ filled phantom decay study and 85 a bolus plus infusion (BI) human neuroreceptor study. Particular focus was given to the bias effects 86 on the values of non-displaceable binding potential $\left(\mathrm{BP}_{\mathrm{ND}}\right)$ at count rate situations normally found 87 in dynamic $\left[{ }^{11} \mathrm{C}\right] \mathrm{ABP} 688(\mathrm{ABP})$ equilibrium PET studies. An alternative method for gathering the 88 PET coincidences into a framing scheme is also proposed in which the counts per frame are kept 89 at the same value for all reconstructed frames of the dynamic acquisition. As anticipated in (12, 90 16), the bias is dependent, in part, on the number of counts in the frame. This leads to the 91 conclusion that the bias may be constant throughout the entire TAC when maintaining constant 92 counts per frame over the entire time interval of the dynamic PET acquisition. Based on this 93 premise, different framing schemes were also compared with respect to bias size and the limits of 94 the bias range that a task must overcome in order to be effectively identified (how much change a 95 task must induce to exceed the bias limits) were evaluated. Two spherical and one background 96 VOIs from an adapted NEMA phantom were analyzed. The phantom had different dimensions, 97 reflecting our $\mathrm{BI}$ protocol with $\mathrm{ABP}$ a glutamatergic receptor ligand with high and low 98 neuroreceptor density regions to evaluate the limitations in binding quantification due to the bias. 99 In addition, the bias obtained with the vendor's 3D OP-OSEM reconstruction was compared with 100 the bias obtained with an in-house reconstruction (PRESTO (17)) both in the phantom study and 101 with human data with ABP. 
102

103

104

105

106

107

108

109

110

111

112

113

114

115

116

117

118

119

120

121

122

123

124

\section{Methods}

\section{A. PET Acquisitions}

All PET data (phantom and human brain studies) were acquired using a 3T hybrid MR-BrainPET insert system (SIEMENS, Erlangen, Germany) in list mode (18). The coincidences were corrected for random events using the delayed window technique with VRR, dead time, attenuation and scattered coincidences (single scatter simulation - SSS method), and physical decay. The image reconstruction was performed with the vendor-supplied 3D OP-OSEM (19) and in-house developed PRESTO implementations (2 subsets and 32 iterations, and 80 iterations respectively), with an isotropic voxel of $1.25 \mathrm{~mm}$ into a volume consisting of 153 transverse slices of $256 \times 256$ pixels. The vendor's reconstruction uses sinograms with span 9 axial data compression, whereas PRESTO is realized as a direct line of response (LOR) reconstruction without LOR data compression. Post-processing was performed with a $2.5 \mathrm{~mm}$ 3D Gaussian filter. Pmod software 3.9 was used to define the VOIs and to extract activity concentration $\left(\mathrm{kBq} / \mathrm{cm}^{3}\right)$ for the analysis.

\section{B. Phantom Study}

An adapted NEMA phantom (20) with sphere inserts filled with ${ }^{11} \mathrm{C}$ was used and coincidence data were acquired during seven isotope half-lives, thus giving 163 minutes total acquisition time for ${ }^{11} \mathrm{C}$ with its half-life $\mathrm{T}_{1 / 2}=20.38$ minutes. An interval of $3 \mathrm{~T}_{1 / 2}$, from 62 minutes to 122 minutes of the acquisition time was used for data analysis. This acquisition time interval was chosen according to the typical count rates measured in a human brain study with ABP alongside a BI protocol (see Section $C$ ) and a scan time of 65 minutes $\left(\sim 3 \mathrm{~T}_{1 / 2}\right.$ of $\left.{ }^{11} \mathrm{C}\right)$. The ratio of the activity concentration between the two spheres (Hot ${ }_{1}$ with $28 \mathrm{~mm}$ of internal diameter and Hot 2 with 22 $\mathrm{mm}$ of internal diameter) and the background region (BG) was $\sim 2: 1$. This value is frequently found for the ratio of activity concentrations in the grey matter cortex (GM) and cerebellar grey matter 
125 (CER, reference region) during the steady-state condition in ABP studies. The activity

TABLE 1

${ }^{11}$ C DECAY EXPERIMENT WITH A PHANTOM (SELECTED TIME INTERVAL)

\begin{tabular}{|c|c|c|}
\hline Time interval in multiples of $\mathbf{T}_{\mathbf{1} / \mathbf{2}}$ & $\begin{array}{c}\text { Phantom Regions } \\
\text { (VOIs names) }\end{array}$ & $\begin{array}{c}\mathbf{1 1}^{\mathbf{1}} \mathbf{C} \text { Activity Concentration } \\
\left(\mathbf{k B q} / \mathbf{c m}^{\mathbf{3}}\right)\end{array}$ \\
\hline \multirow{2}{*}{ T4 } & Hot1/Hot2 & 15.30 \\
& BG & 8.25 \\
\hline \multirow{2}{*}{ T5 } & Hot1/Hot2 & 7.65 \\
& BG & 4.13 \\
\hline \multirow{2}{*}{ T6 } & Hot1/Hot2 & 3.82 \\
& BG & 2.06 \\
\hline
\end{tabular}

The half-life here is expressed as the number of elapsed ${ }^{11} \mathrm{C}$ half-lives during the decay experiment, where $\mathrm{T} 4$ is during the fourth $\mathrm{T}_{1 / 2}\left({ }^{11} \mathrm{C}\right)$ and so forth. The chosen $\mathrm{T} 4, \mathrm{~T} 5$ and $\mathrm{T} 6$ were selected to represent typical activity concentration in human $\left[{ }^{11} \mathrm{C}\right] \mathrm{ABP} 688$ acquisitions.

A cold transmission scan of the adapted NEMA phantom using ${ }^{68} \mathrm{Ge}$ sources was acquired in a Siemens ECAT Exact HR+ PET scanner. This acquisition (2 bed positions with 20 minutes of transmission in a $256 \times 256$ matrix and reconstructed with OSEM 2D -6 iterations and 16 subsets) was used to create the attenuation map for the phantom used in the ${ }^{11} \mathrm{C}$ decay study.

To obtain the ground truth data for the phantom study, the activity concentrations in the different phantom compartments were measured with a gamma counter (Wizard counter) repeating for 3 probes in a solution of $0.5 \mathrm{ml}$. Decay correction, counter calibration factor and volumes/weights of the probes were considered and standard corrections applied.

Figure 1 shows a PET image from ABP overlaid with an MR T1 image acquisition and a comparison to the image of an adapted NEMA phantom. 
a)

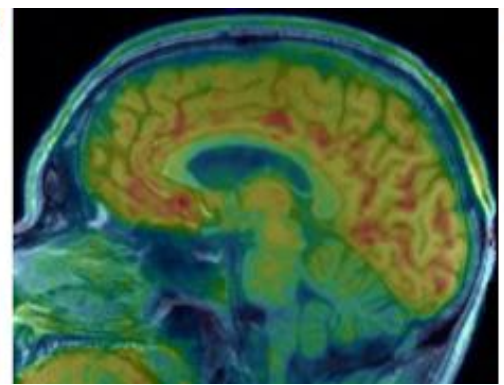

b)

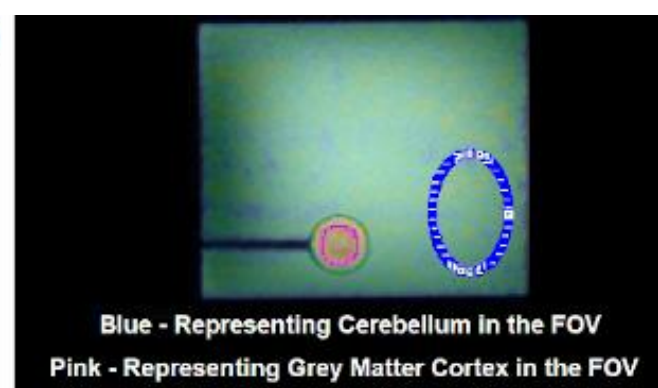

Fig. 1. a) Sagittal PET image from the ABP study overlaid with MR T1 image and b) Sagittal PET image of the NEMA phantom overlaid with an MR T1 image during the ${ }^{11} \mathrm{C}$ filled phantom decay study. The high activity concentration region (pink VOI) and low activity concentration region (blue VOI) is highlighted for bias analysis.

Based on the known neuroreceptor distribution of ABP, the background VOI (BG) was considered to be representative of CER as a reference region and the two hot sphere's VOIs (Hot 1 and $\mathrm{Hot}_{2}$ ) were considered to be representative of the GM regions, i.e. parts of the cingulate cortex (neuroreceptor rich region in $\mathrm{ABP}$, see Section $C$ ) and the nucleus accumbens. The latter is important for the analysis of psychiatric conditions, such as schizophrenia (21). The effective volumes of the three VOIs were: $112.86 \mathrm{ml}$ (BG, 57782 voxels), $3.42 \mathrm{ml}$ (Hot ${ }_{1}, 1751$ voxels), and $1.13 \mathrm{ml}\left(\mathrm{Hot}_{2}, 578\right.$ voxels) respectively. To reduce partial volume effects in the analysis, the VOIs were drawn with a distance of $4 \mathrm{~mm}$ to the inner borders of the $\mathrm{Hot}_{1}$ and $\mathrm{Hot}_{2}$ spheres (effective internal diameters of $20 \mathrm{~mm}$ and $14 \mathrm{~mm}$, respectively). Moreover, in the ${ }^{11} \mathrm{C}$ filled phantom decay study, following the application of all data corrections, a constant activity concentration was expected during the entire acquisition time (T1 to T7) in all phantom compartments since a BI study at equilibrium conditions aims to maintain constant activity concentrations in the brain compartments.

\section{Human Study with $\left[{ }^{11}\right.$ C]ABP688}

PET Protocol with $\left[{ }^{11}\right.$ C]ABP688: An analysis similar to that used in the phantom study was applied to already existing data from a dynamic PET acquisition on a subject with $437 \mathrm{MBq}$ of totally administered activity of ABP. Details of this study can be found in (22). The radiosynthesis 
168 of ABP was performed according to (23).

169 Applied BI protocol: The bolus injection had 50\% of the total injected activity, followed by 65

170 minutes of infusion with $92 \mathrm{ml} / \mathrm{h}$. The bolus injection was applied after positioning the subject in

171 the scanner and began simultaneously with the start of PET data acquisition. A distribution

172 equilibrium was observed at around 30 minutes after the bolus injection, and PET data acquisition

173 stopped at 65 minutes with the end of the infusion. The emission data were corrected for

174 attenuation using template-based methods (24). Head motion was corrected with a frame-by-frame

175 realignment to a reference image (frame length 5 minutes post-injection) and was performed with

176 Pmod 3.9. Head motion was lower than $1 \mathrm{~mm}$, which is less than the PET spatial resolution of

$1773 \mathrm{~mm}$ at the center of the FOV. VOIs were selected in CER, anterior cingulate cortex (ACC) and

178 posterior temporal cortex (Post-Tl), as the last two regions have a high density of metabotropic

179 glutamatergic receptors type 5 (mGluR5) (25).

\section{Reconstruction - Frame Schemes}

181 The different framing schemes used to test bias in the time activity curves (TACs) and binding 182 potential values were defined by either constant or increasing frame lengths, as well as an 183 alternative framing scheme with variable frame lengths that takes the decreasing count rate during 184 the dynamic PET into account. The framing schemes for the phantom study and the human brain 185 study were defined as follows:

186 Constant Frame Length Schemes (Const): PET list-mode data of the entire acquisition was sorted 187 into time frames with constant frame lengths of 2, 3 or 5 minutes respectively (Const 2 min, Const $1883 \mathrm{~min}$ and Const $5 \mathrm{~min}$ ).

189 Increasing Frame Length Scheme (Incr): PET list-mode data was sorted into time frames with 190 increasing frame length, i.e. during T4 the frame length was set to 2 minutes and during T5 and 
191 T6 the frame length was set to 3 minutes and 5 minutes (Incr 2-3-5 min), respectively. In this way,

192 the lower counts for later frames caused by the radioactive decay of ${ }^{11} \mathrm{C}$ was compensated to some 193 extent.

194 Increasing Frame Length Scheme with Constant True Counts (Const Trues): True events versus 195 time curves were extracted from the acquisition and the frame lengths were adjusted to values 196 which yielded the same total counts per frame for all frames of the dynamic PET data. The number 197 of counts in the final frame of the acquisition was taken as a reference for counts per frame. Earlier 198 frames were accordingly shorter. A duration of 5 minutes was chosen for the final frame since this 199 is a typical setting for applications with cognitive tasks in simultaneous PET/MR applications and 200 our BI protocol. The three framing schemes were evaluated with both reconstructions.

201 We would like to emphasize here that the advantage of the BI protocol in our case is the simplicity 202 over the dual-bolus injection approach, because it allows us to measure the baseline and challenge 203 effects in $\mathrm{BP}_{\mathrm{ND}}$ in a single acquisition session. Another important point is the simultaneous use of 204 other imaging or monitoring modalities, such as magnetic resonance (MR) or 205 electroencephalography (EEG) at the same brain state condition. Our presented approach can also 206 be applied to pure bolus or pure infusion protocols since the bias in estimated binding values, is 207 not caused by the activity application protocol, but the image reconstruction itself. However, the 208 bias reduction for parameters estimated with kinetic modeling still needs to be evaluated in an 209 follow up study.

\section{E. Bias Analysis}

211 Bias and variability were analyzed as follows: 
212 Activity Concentration Accuracy: The bias of the measured activity concentration ( $\left.\mathrm{A}_{\text {measured }}\right)$ was

213 computed by (1):

$$
\text { Bias }[\%]=\left[\frac{\left(A_{\text {measured }}-A_{\text {true }}\right)}{A_{\text {true }}}\right] \times 100
$$

215 where $A_{\text {true }}$ is the true activity concentration from the probes measured in the gamma counter 216 (ground-truth value) and $\mathrm{A}_{\text {measured }}$ is the mean activity concentration in the VOIs representing the 217 cerebellum (blue VOI in Figure1(b)) or hot spheres (pink Hot $\mathrm{H}_{1}$ VOI as an example in Figure1(b)) 218 in each frame. PRESTO was cross-calibrated to the vendor's 3D OP-OSEM reconstruction with 219 respect to the activity concentration accuracy. Box plots were used to represent the bias variability 220 of the measurements for each VOI, for the different acquisition intervals T4, T5, T6, and for the 221 different framing schemes.

222 Binding Potential Accuracy: The procedure described above in (1) was also used to estimate the 223 bias and variability of the binding potential values (BP), by considering BP instead of the activity 224 concentration $\mathrm{A}$. The $\mathrm{BP}_{\text {true }}\left(\right.$ for $\mathrm{Hot}_{1}$ and $\mathrm{Hot}_{2}$ ) values were estimated as presented in (2). Equation 2253 was used to calculate $\mathrm{BP}_{\text {measured. }}$

$$
\mathrm{BP}_{\text {True }}=\left[\frac{A_{\text {TrueHot }}}{A_{\text {TrueBG }}}\right]-1
$$

$$
\mathrm{BP}_{\text {Meaured }}=\left[\frac{A_{V O I 1}}{A_{V O I 2}}\right]-1
$$

229 where AvoI1 is the mean activity concentration in the $\mathrm{VOI}_{1}$ (high activity concentration region $230 \mathrm{Hot}_{1}$ or $\mathrm{Hot}_{2}$ for the phantom and ACC or Post_Tl for human data) and Avor2 is the mean activity 231 concentration in the $\mathrm{VOI}_{2}$ (low activity concentration region - representing $\mathrm{BG}$ for the phantom 232 or CER for human data). Again, box plots were used to represent the variability of the BP values 
233 for the different framing schemes, time intervals and regions. BP was used for the phantom study 234 and $\mathrm{BP}_{\mathrm{ND}}$ was used for the human study (binding potential values).

235 Linear Fit and Slope: TACs recorded in the phantom and human brain studies were also 236 analyzed by evaluating the slope of a straight line fitted to the data. The percentage change per 237 hour $[\% / \mathrm{h}]$ of the TACs were evaluated for the equilibrium interval (human study) or the time 238 intervals T4, T5 and T6 (phantom study) and compared. By choosing the Const Trues framing 239 scheme, we hypothesized that the same bias would always be obtained throughout the TACs. Thus, 240 the bias should not change the slope of the TACs. However, bias from framing schemes that do 241 not consider constant true counts per frame, such as Const or Incr schemes, may show different 242 slope values between the time intervals and higher slope values in the human data study.

243 Standard error (SE): Statistical SE was computed according to (4) and Gaussian error 244 propagation was used to estimate the SE for the BP values.

245

$$
\mathrm{SE}[\%]=\left[\frac{\sigma}{\sqrt{n}}\right] \times 100
$$

246 where $\sigma$ is the standard deviation in the VOI regions and $n$ is the number of pixels from the VOI.

247 This analysis was performed for both reconstructions. 


\section{Results}

\section{A. Phantom Study}

Activity Concentration Accuracy: Figure 2 shows the relative bias for the three regions in explained in Section D.
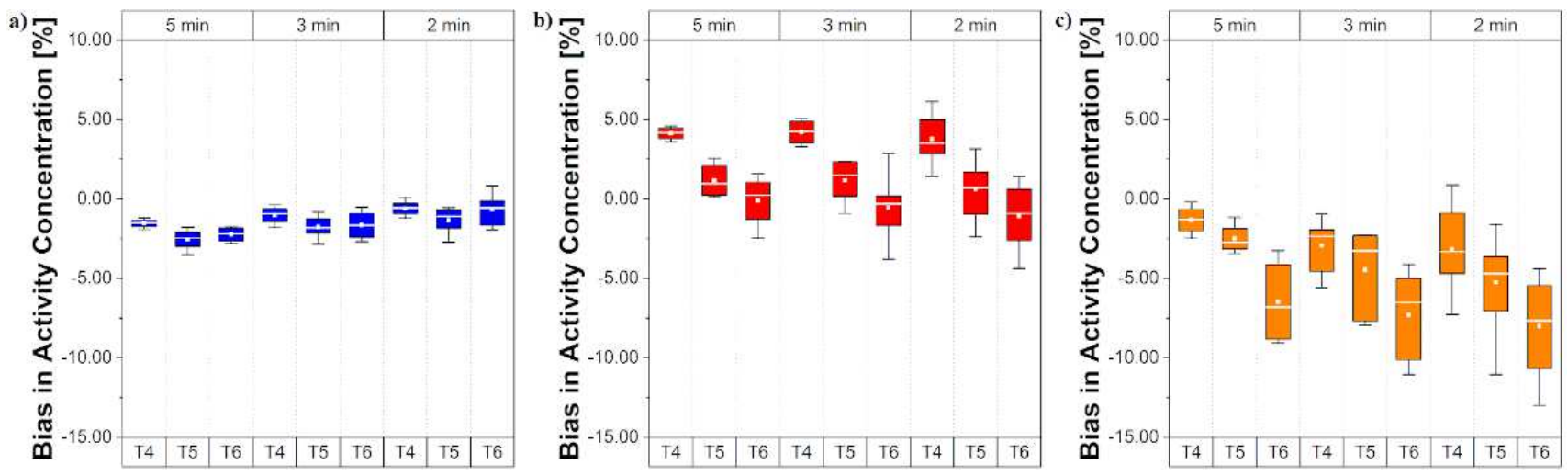

Fig. 2. Bias and variability in activity concentration for a) BG region (low activity concentration), b) $\mathrm{Hot}_{1}$ region (high activity concentration) and c) $\mathrm{Hot}_{2}$ region (high activity concentration); all with vendor's reconstruction.

In Figure 2 a) it is possible to notice a slightly increased bias in the BG region with decreasing 

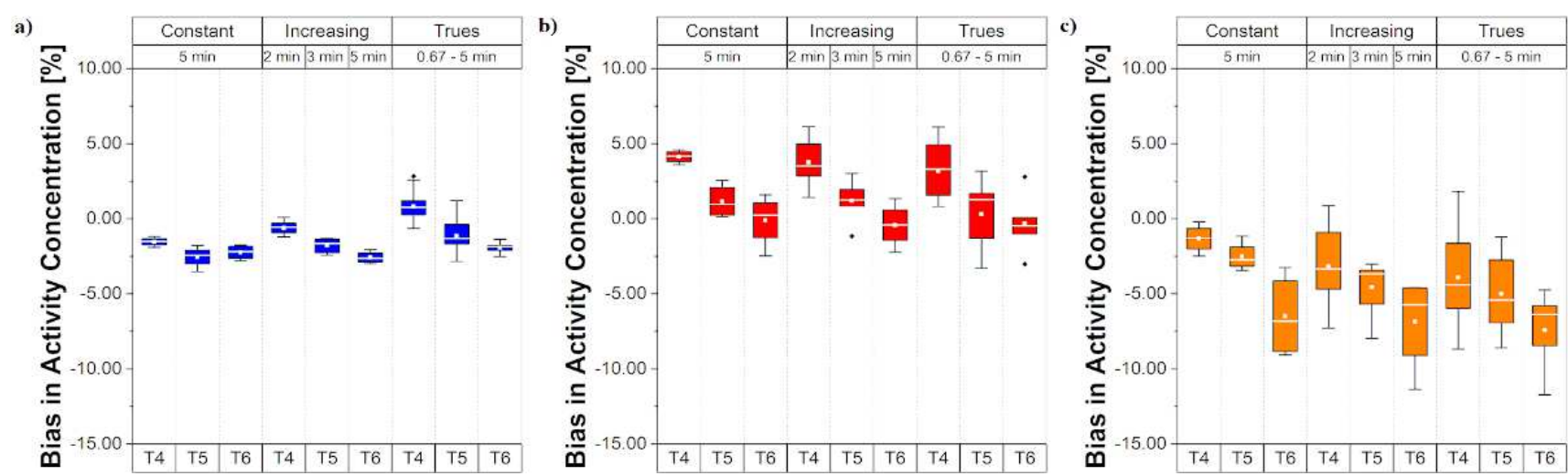

Fig. 3. Bias and variability in activity concentration for different framing schemes a) BG region (low activity concentration), b) Hot 1 region (high activity concentration) and c) $\mathrm{Hot}_{2}$ region (high activity concentration); all with vendor's 3D OP-OSEM reconstruction.

A similar trend for the $\mathrm{BG}$ region can be noticed in terms of increased bias with decreasing frame length, but this time with reduced bias in T6 and the opposite behavior for Incr and Const Trues schemes is seen. In the hot regions, the negative bias is maintained, but again there is a reduced bias in the T6 time interval for Incr and Const Trues schemes compared to Const schemes, especially with the Const Trues scheme.

\section{Binding Potential Accuracy:}

Figure 4 presents the relative errors of BP values in the two hot phantom VOIs for different framing schemes and time intervals.
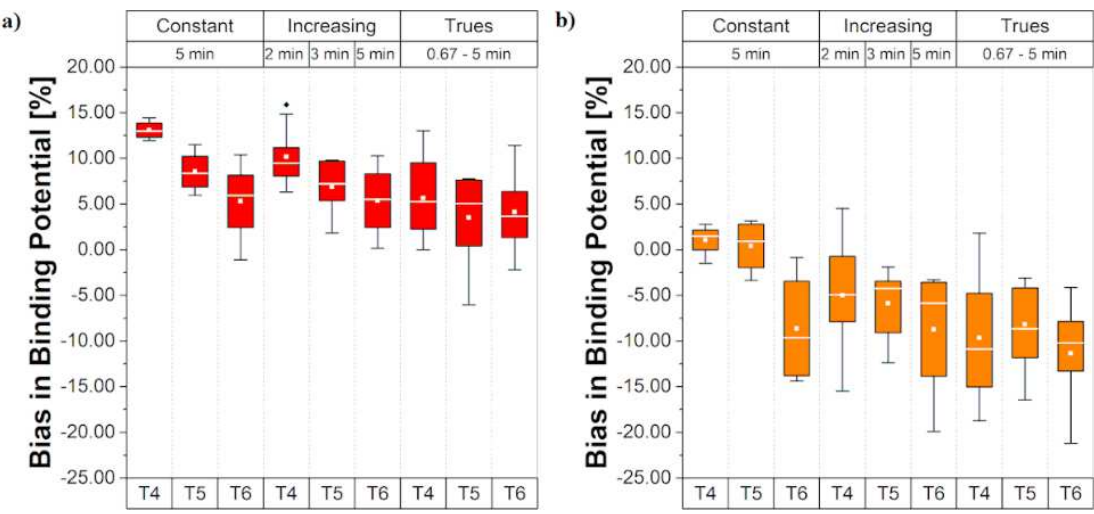

Fig. 4. Bias and variability of BP values for a) $\mathrm{Hot}_{1}$ region, and b) $\mathrm{Hot}_{2}$ region; all with vendor's 
Note that, for the scheme with Const 5 min framing, the bias became variable within the time interval (from $12 \%$ to $5 \%$ for $\mathrm{Hot}_{1}$ and from $1 \%$ to $-10 \%$ for $\mathrm{Hot}_{2}$ ), suggesting a different bias range according to the time intervals during the acquisition. This trend is minimized when Incr 23-5 min and Const Trues framing schemes were used and the bias range remained even closer in the all-time interval with the Const Trues scheme (Figure 4 a) bias around 5\% in all-time interval and b) bias around $-10 \%$ in all-time interval). However, in the $\mathrm{Hot}_{2}$ region, the bias became higher negative and with higher variability, especially in the T6 interval for all schemes tested (up to - 10\%) even with the proposed framing Const Trues. It is important to bear in mind that these data were not corrected for partial volume effects (PVE). However, the maximum estimated effect is around $15 \%-17 \%$ and the estimated correction factor is around 0.84 for the reported activity concentration values (hot regions are $4 \mathrm{~cm}$ far away from the FOV center). The PVE can be expected to be the same size for all framing schemes and time intervals. PVE was estimated with a geometric transfer matrix (GTM) using Pmod.

\section{Time Activity Curves and Fit Analysis:}

As shown in Figure 5 for four different framing schemes, the TACs of VOIs (BG and Hot $_{1}$ ) were approximated separately by individual linear fits for the intervals T4, T5 and T6. Hot 2 will no longer be shown since a larger bias and variability for quantification was noticed in this region. The BP values became particularly unreliable and we do not intend to analyze such small region in our BI protocol in the future due to the complexity of task effects analysis. A similar trend found in Figure 5. can be seen in the box plots (Figure 2 and Figure 3), which show a negative slope for the BG region in T4 and T5 and positive slopes in T6 (more pronounced in Const schemes). Note the negative slopes in the $\mathrm{Hot}_{1}$ region for different framing scheme methods. Slope values $\pm \mathrm{SE}$ for the decay corrected TACs are outlined in Table 2. 
a)
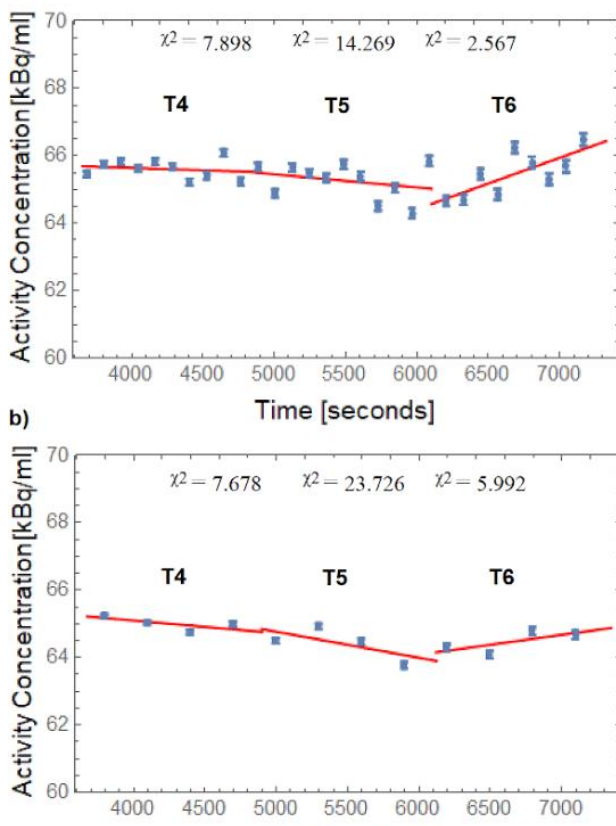

c)
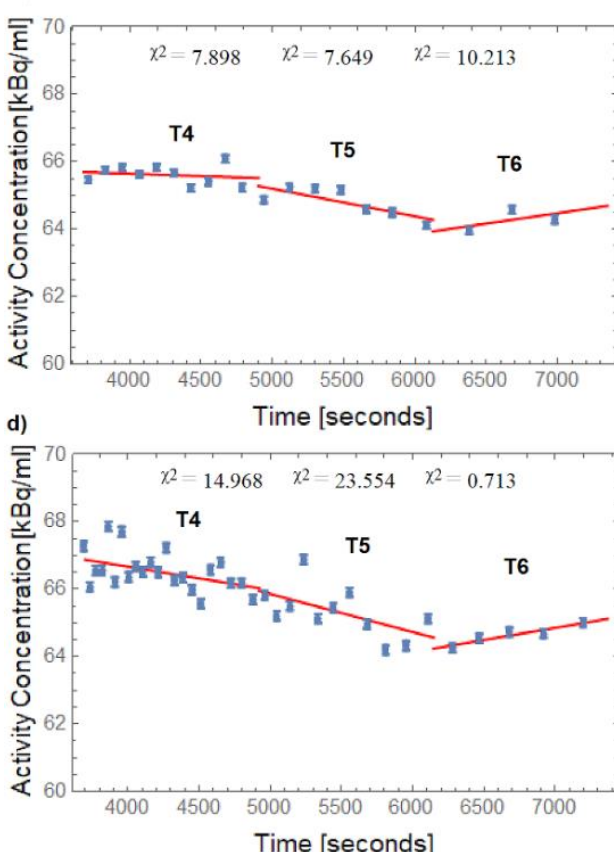
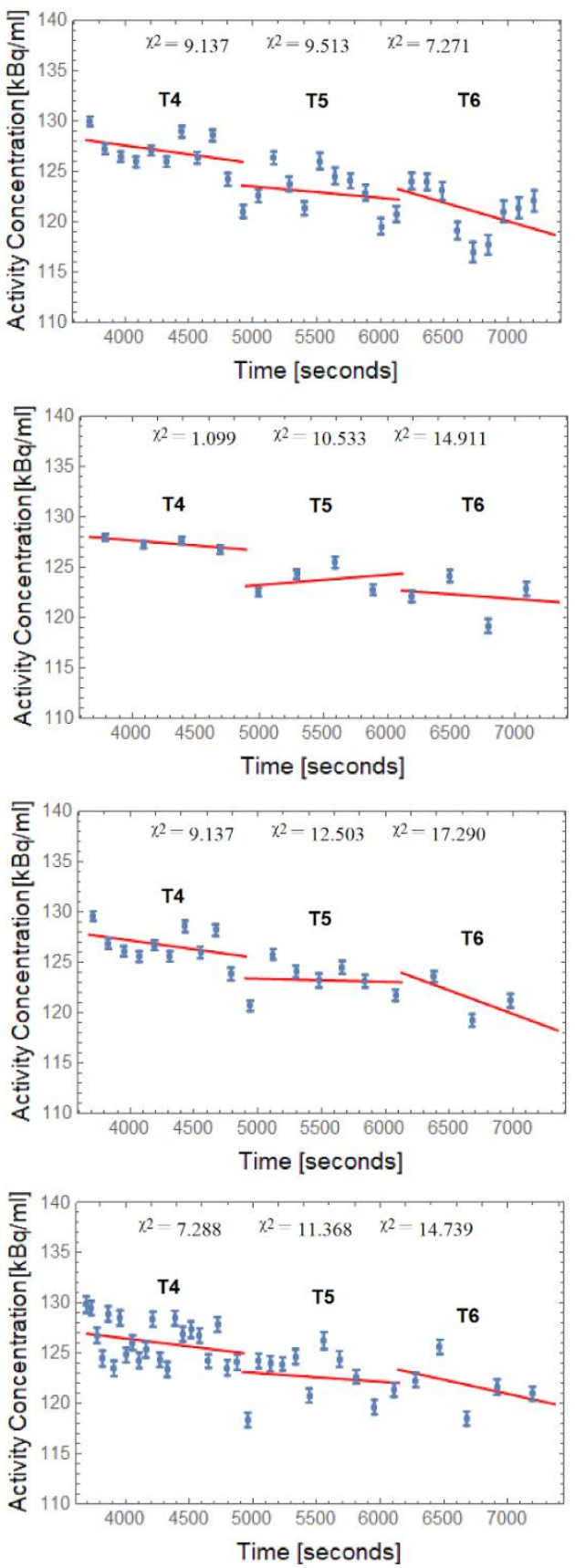

Fig. 5. TACs and linear fits for BG (left side) and Hot (right side) regions for a) Const $2 \mathrm{~min}$, b) Const 5min, c) Incr 2-3-5 min and d) Const Trues; all with vendor's 3D OP-OSEM reconstruction. $\chi^{2}$ : goodness of linear fit. 
TABLE 2

TIME ACTIVITY CURVES - SLOPE* RESUlTS - PHANTOM DATA

\begin{tabular}{|c|c|c|c|}
\hline Frame Scheme & Half-Life $\left(T_{1 / 2}\right)$ & $\begin{array}{c}\text { Slope } \pm \text { SE }(\% / h) \\
\text { BG }\end{array}$ & $\begin{array}{c}\text { Slope } \pm \text { SE }(\% / h) \\
\text { Hot1 }\end{array}$ \\
\hline \multirow{3}{*}{ Const 2 min } & $\mathrm{T} 4$ & $-0.8 \pm 1.4$ & $-5.0 \pm 4.3$ \\
\hline & T5 & $-2.1 \pm 2.2$ & $-3.4 \pm 5.2$ \\
\hline & T6 & $8.4 \pm 2.7$ & $-11.1 \pm 8.1$ \\
\hline \multirow{3}{*}{ Const 5 min } & $\mathrm{T} 4$ & $-2.1 \pm 1.5$ & $-2.9 \pm 1.7$ \\
\hline & T5 & $-4.3 \pm 3.5$ & $2.9 \pm 6.7$ \\
\hline & T6 & $3.3 \pm 2.3$ & $-2.8 \pm 10.7$ \\
\hline \multirow{3}{*}{ Incr $2-3-5$ min } & $\mathrm{T} 4$ & $-0.8 \pm 1.4$ & $-5.0 \pm 4.3$ \\
\hline & T5 & $-4.5 \pm 1.6$ & $-0.9 \pm 5.7$ \\
\hline & T6 & $3.5 \pm 4.8$ & $-13.9 \pm 17.3$ \\
\hline \multirow{3}{*}{ Const Trues } & $\mathrm{T} 4$ & $-3.7 \pm 1.8$ & $-4.5 \pm 3.6$ \\
\hline & T5 & $-6.2 \pm 2.9$ & $-2.6 \pm 5.9$ \\
\hline & T6 & $4.0 \pm 0.8$ & $-8.3 \pm 11.1$ \\
\hline
\end{tabular}

*Slope - Linear fits from graphs presented in Figure 5, and SE - Standard Error.

For human brain studies, the true activity concentrations in brain regions and the binding potential values are not known. Therefore, here, the TACs during the equilibrium phase of the

322 acquisition were directly analyzed using linear fits (Figure 6) to compare the reconstruction bias of the different framing schemes. Table 3 shows the corresponding slope results $(\% / \mathrm{h} \pm \mathrm{SE})$. 

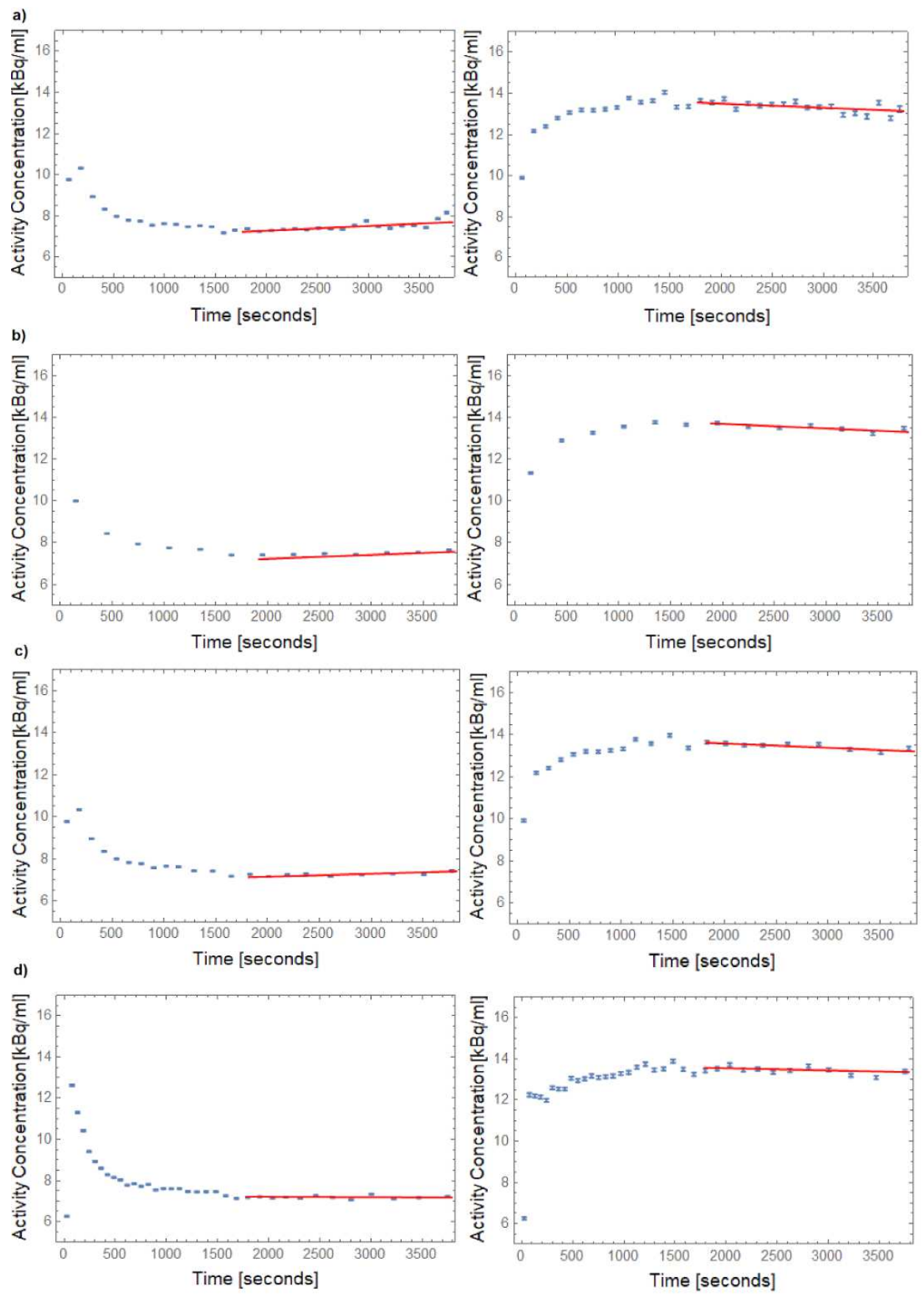

Fig. 6. TACs and linear fits during equilibrium in CER (left side) and ACC (right side) regions from a human brain study for a) Const 2 min, b) Const 5 min, c) Incr 2-3-5 min and d) Const Trues; all with vendor's 3D OP-OSEM reconstruction. 
TABLE 3

TIME ACTIVITY CURVES - SLOPE* RESUlTS - HuMAN DATA

\begin{tabular}{|c|c|c|}
\hline Frame Scheme & $\begin{array}{c}\text { Slope } \pm \text { SE }(\% / h) \\
\text { CER }\end{array}$ & $\begin{array}{c}\text { Slope } \pm \text { SE }(\% / h) \\
\text { ACC }\end{array}$ \\
\hline Const 2 min & $12.3 \pm 2.8$ & $-7.7 \pm 2.0$ \\
\hline Const 5 min & $5.3 \pm 1.0$ & $-4.7 \pm 1.7$ \\
\hline Incr 2-3-5 min & $3.4 \pm 1.6$ & $-5.1 \pm 1.1$ \\
\hline Const Trues & $0.6 \pm 1.9$ & $-4.9 \pm 1.9$ \\
\hline
\end{tabular}

*Slope - Linear fits from graphs presented in Figure 6, and SE - Standard Error.

Using the human brain data, it is again possible to confirm the bias behavior for low activity concentration regions as CER and high activity concentration regions as ACC (see Figure 6 and Table 3). Please note the higher slope values in CER when using Const framing methods and its reduction when applying the proposed Const Trues framing scheme method (results using vendor's reconstruction).

BP values with $\left[{ }^{11} C\right] A B P 688:$

The effects of the different framing schemes on $\mathrm{BP}_{\mathrm{ND}}$ values are shown in Figure 7. Here 49 the effects of bias propagation into $\mathrm{BP}_{\mathrm{ND}}$ values is clearly noticeable, especially after 3000 seconds 50 for the Cont 2 min framing schemes. Note the reduction in the bias when Incr 2-3-5 min and Const Trues time length schemes were used. 
a)
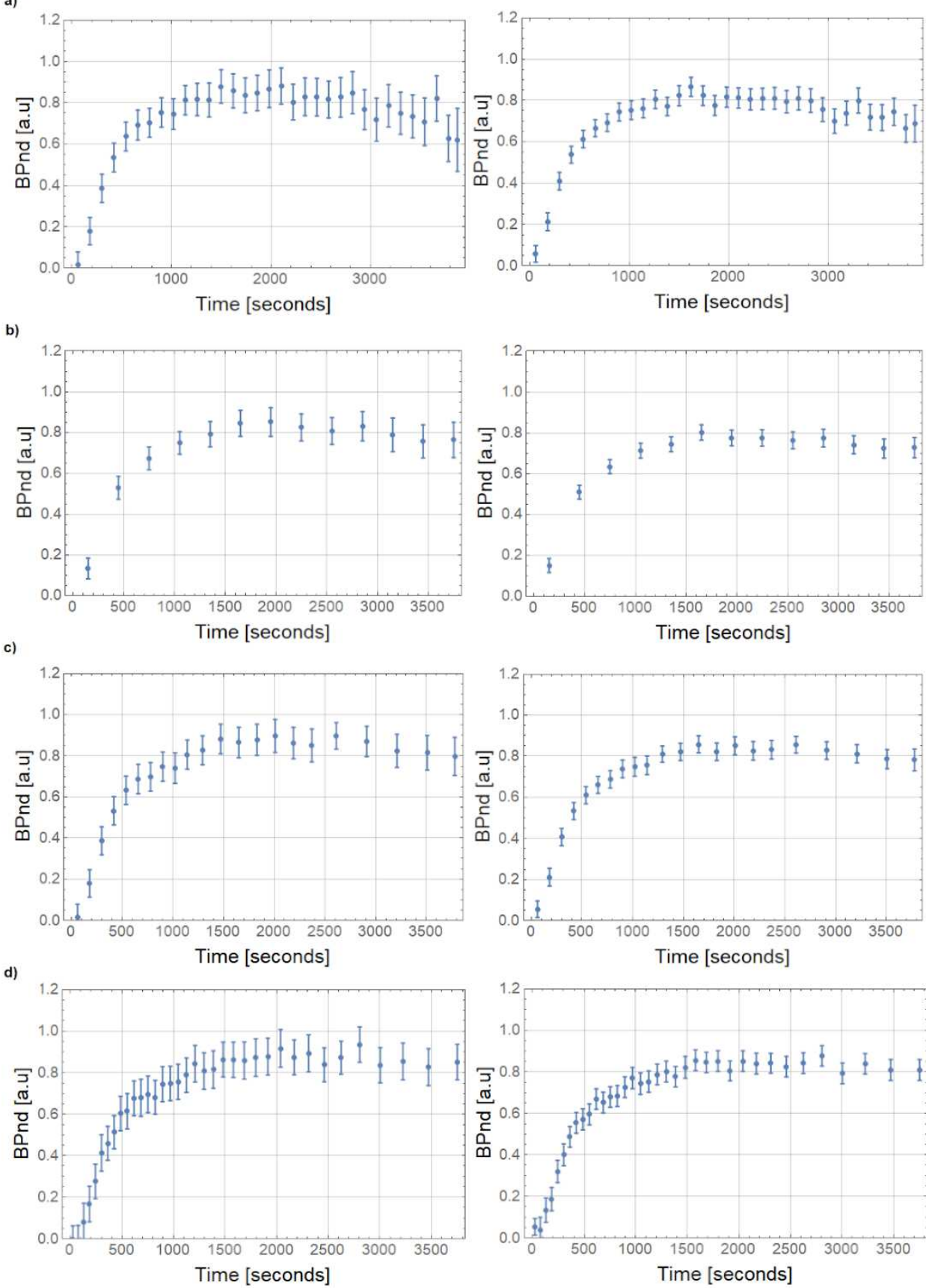
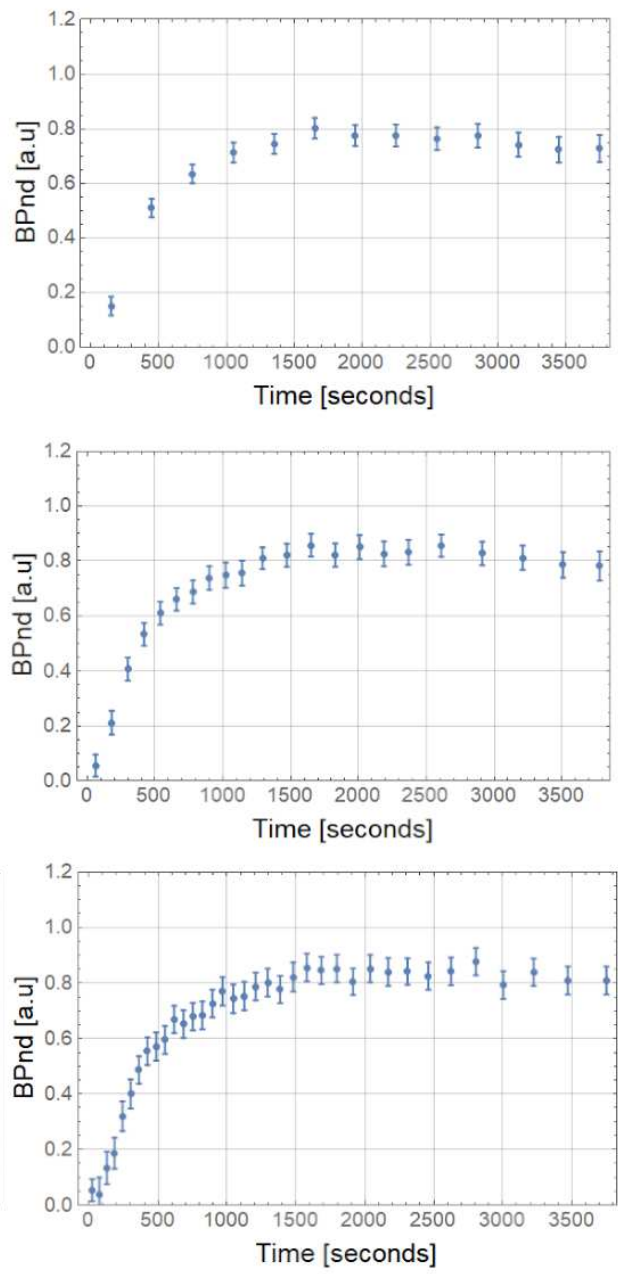

Fig. 7. Values of $\mathrm{BP}_{\mathrm{ND}} \pm \mathrm{SE}$ for $\mathrm{ACC}$ (left side) and Post-Tl (right side) in a human brain study 
For simplicity, the different framing schemes for each reconstruction procedure are shown for comparison in Figure 8.

362

In the comparison between both reconstruction procedures, PRESTO shows no significant rising behavior in the T6 time interval for BG in the Const 5 min framing scheme (see Figure 8.a)). For the Hot $_{1}$ region (Figure 8.b)), the bias is higher, but it is possible to notice that the mean biases are closer to each other for T5 and T6 time intervals, and the spread of the mean bias values from the vendor's reconstruction is slightly higher in the same time interval. Similar behavior is also shown in the BP bias values, but this time when the Const Trues framing scheme is applied, the vendor's reconstruction shows lower mean bias values (around 5\%) during the entire time interval (T4, T5, and T6) against 10\% from PRESTO.

a)

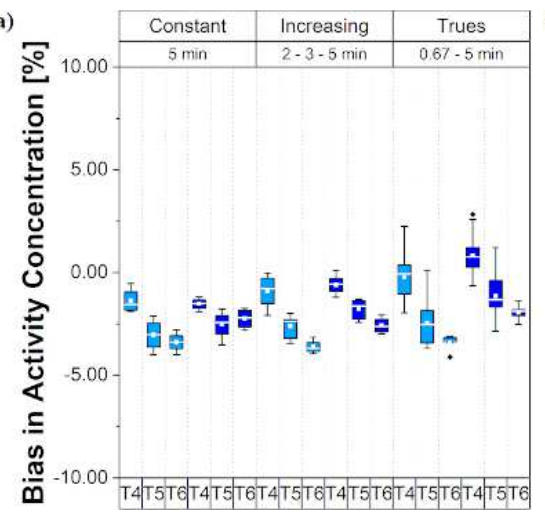

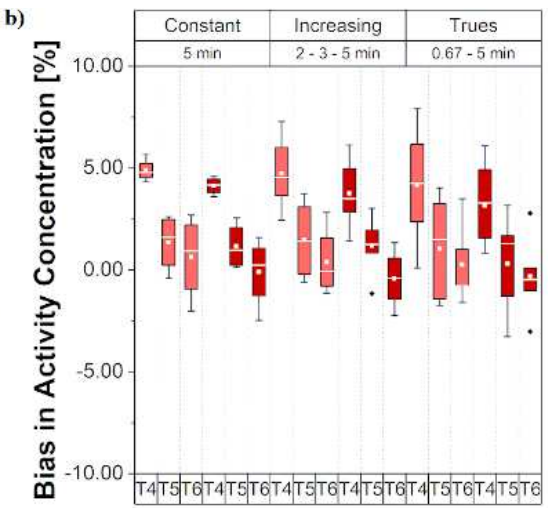

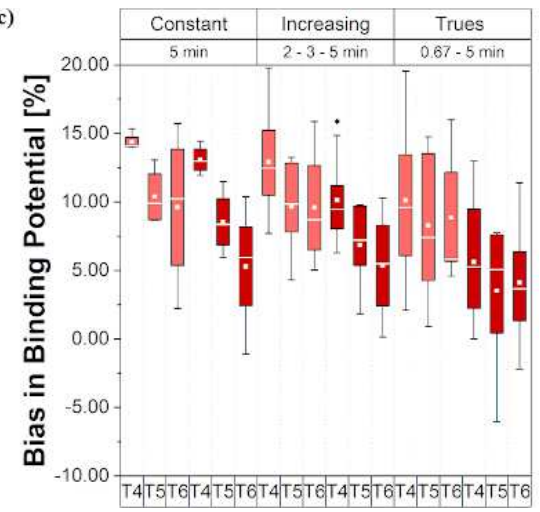

370

371

372

373

374

375

376

377

Fig. 8. Bias in activity concentrations and $\mathrm{BP}$ values for different framing schemes comparing PRESTO (light blue, light red) and vendor's 3D OP-OSEM (dark blue, dark red) in a) BG, b) Hot $_{1}$ and c) Hot HP. $_{1}$

\section{Time Activity Curves and Fit Analysis:}

Slope values for the different framing schemes with PRESTO reconstruction are presented in Table 4, per half-life and phantom regions as shown in Figure 6 and Table 2 for comparison with 
378 vendor's 3D OP-OSEM reconstruction. TACs can be seen in the supplementary file ${ }^{1}$

379

380
TABLE 4

TIME ACTIVITY CURVES - SLOPE* RESUlTS - PhANTOM DATA - PRESTO

\begin{tabular}{|c|c|c|c|}
\hline Frame Scheme & $\begin{array}{c}\text { Half-Life } \\
\left(\mathbf{T}_{1 / 2}\right)\end{array}$ & $\begin{array}{c}\text { Slope } \pm \text { SE }(\% / h) \\
\text { BG }\end{array}$ & $\begin{array}{c}\text { Slope } \pm \text { SE }(\% / h) \\
\text { Hot }_{1}\end{array}$ \\
\hline \multirow{3}{*}{ Const $2 \mathrm{~min}$} & $\mathrm{~T} 4$ & $-5.0 \pm 1.6$ & $-5.8 \pm 5.5$ \\
\hline & $\mathrm{T} 5$ & $-4.2 \pm 2.2$ & $-8.4 \pm 5.8$ \\
\hline & T6 & $4.7 \pm 2.7$ & $-12.7 \pm 9.4$ \\
\hline \multirow{3}{*}{ Const $5 \mathrm{~min}$} & $\mathrm{~T} 4$ & $-5.5 \pm 1.8$ & $-4.8 \pm 1.4$ \\
\hline & T5 & $-5.2 \pm 3.6$ & $-3.7 \pm 8.3$ \\
\hline & T6 & $1.7 \pm 3.2$ & $-0.12 \pm 13.3$ \\
\hline \multirow{3}{*}{ Incr $2-3-5 \mathrm{~min}$} & $\mathrm{~T} 4$ & $-5.0 \pm 1.6$ & $-5.7 \pm 5.5$ \\
\hline & $\mathrm{T} 5$ & $-5.5 \pm 1.1$ & $-5.7 \pm 6.3$ \\
\hline & T6 & $2.2 \pm 4.6$ & $-17.0 \pm 17.8$ \\
\hline \multirow{3}{*}{ Const Trues } & $\mathrm{T} 4$ & $-6.0 \pm 1.8$ & $-7.6 \pm 4.7$ \\
\hline & T5 & $-5.8 \pm 3.0$ & $-10.5 \pm 6.0$ \\
\hline & T6 & $3.5 \pm 1.4$ & $-12.2 \pm 9.2$ \\
\hline
\end{tabular}

381

382

383

384

385

386

387

388

\section{Human Study:}

TACs Fit Analysis with $\left[{ }^{11}\right.$ C]ABP688:

The evaluation explained in Section $D$ was repeated for the PRESTO reconstruction and the results are given in Figure 9 and Table 5.

\footnotetext{
${ }^{1}$ PRESTO TACs and linear fits for BG, Hot 1 regions and $x^{2}$ goodness of linear fit are available in supplement.
} 

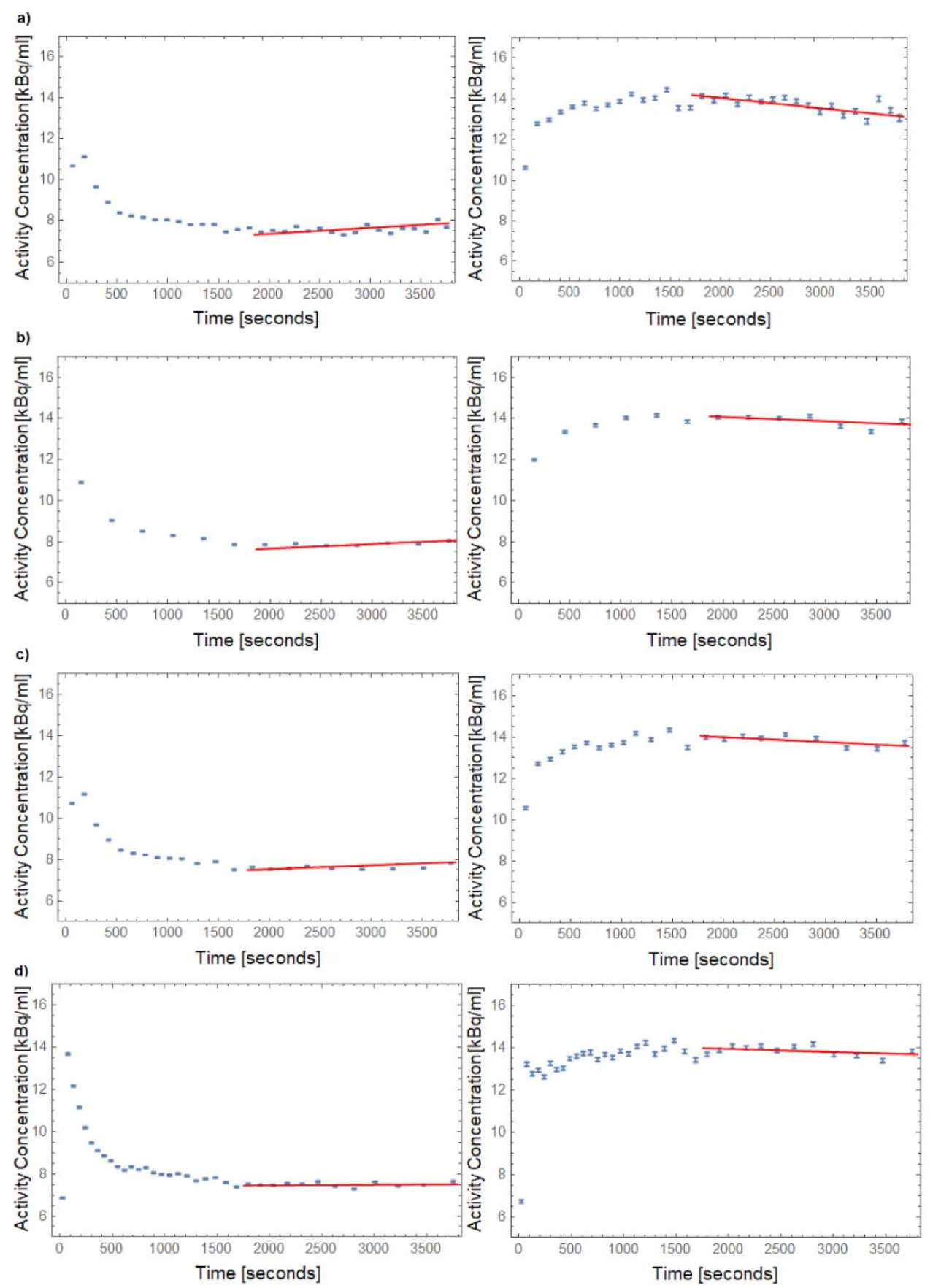

Fig. 9. TACs and linear fits during equilibrium in CER (left side) and ACC (right side) regions from a human brain study for a) Const $2 \mathrm{~min}$, b) Const $5 \mathrm{~min}$, c) Incr 2-3-5 min and d) Const 
401

402

403

404 Comparisons between the vendor's 3D OP-OSEM reconstruction (see Figure 6 and Table 3) and 405 406 407 408

409 410
TABLE 5

TIME ACTIVITY CURVES - SLOPE* RESUlTS - HuMAN DATA - PRESTO

\begin{tabular}{|c|c|c|}
\hline Frame Scheme & $\begin{array}{c}\text { Slope } \pm \text { SE }(\% / h) \\
\text { CER }\end{array}$ & $\begin{array}{c}\text { Slope } \pm \text { SE }(\% / h) \\
\text { ACC }\end{array}$ \\
\hline Const 2 min & $4.0 \pm 3.0$ & $-11.6 \pm 2.6$ \\
\hline Const 5 min & $3.4 \pm 1.8$ & $-7.4 \pm 3.2$ \\
\hline Incr 2-3-5 min & $2.9 \pm 2.1$ & $-6.7 \pm 2.2$ \\
\hline Const Trues & $1.5 \pm 2.5$ & $-6.2 \pm 2.4$ \\
\hline
\end{tabular}

*Slope - Linear fits from graphs presented in Figure 9, and SE - Standard Error.
PRESTO are possible by looking at the slope values in BG and CER low activity concentration regions. As already shown in the box plots (Figure 8 a)), it is possible to achieve lower slope values in these regions using PRESTO reconstruction. However, higher bias in $\mathrm{Hot}_{1}$ and ACC regions was also shown for PRESTO.

BP $P_{N D}$ values with $\left[{ }^{11} C\right] A B P 688$ :

11 Figure 10 presents $\mathrm{BP}_{\mathrm{ND}}$ values using PRESTO reconstruction and Table 6 shows the slope from 12 the $\mathrm{BP}_{\mathrm{ND}}$ curves for the different framing schemes and for both reconstruction procedures during the equilibrium phase (from 1800 seconds to the acquisition end). 

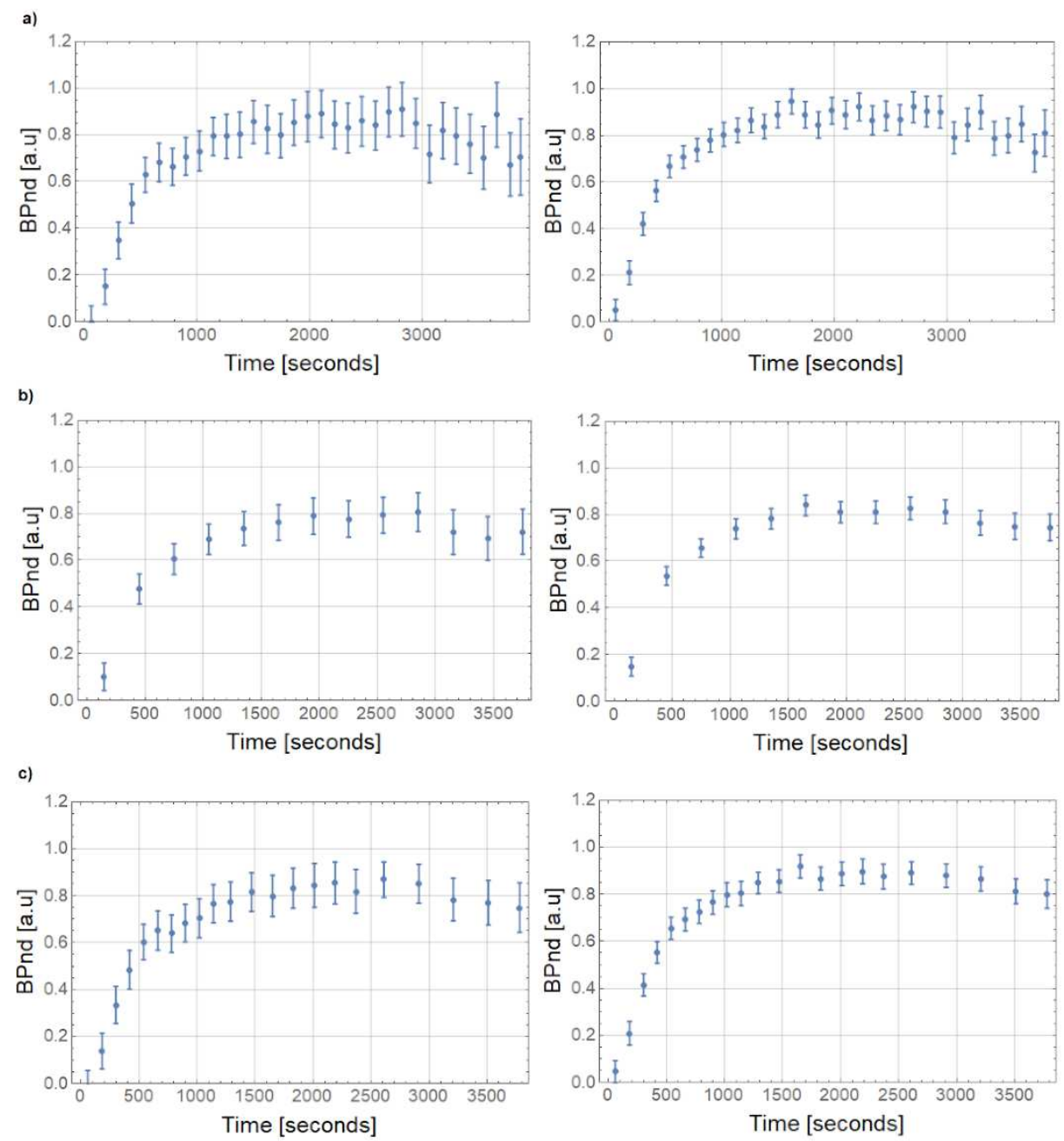

d)
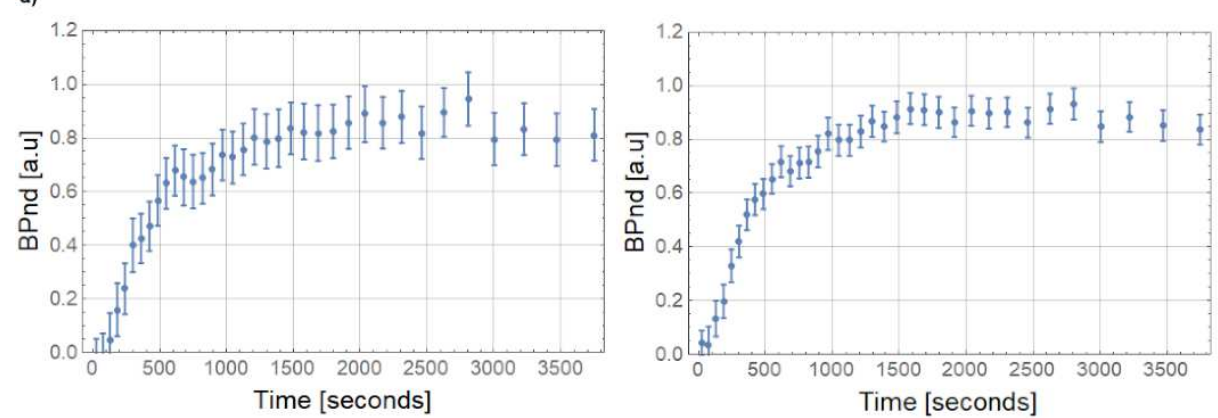

Fig. 10. Values of $\mathrm{BP}_{\mathrm{ND}} \pm \mathrm{SE}$ values for $\mathrm{ACC}$ (left side) and Post-Tl (right side) regions from a human brain study for a) Const $2 \mathrm{~min}$, b) Const $5 \mathrm{~min}$, c) Incr 2-3-5 min and d) Const Trues; all with PRESTO reconstruction. 
TABLE 6

BP $_{\text {ND }}$ CURVES SLOPES* RESULTS - HUMAN DATA - VENDOR'S 3D OP-OSEM

\begin{tabular}{|c|c|c|}
\hline Frame Scheme & $\begin{array}{c}\text { Slope } \pm \text { SE }(\% / h) \\
\text { ACC }\end{array}$ & $\begin{array}{c}\text { Slope } \pm \text { SE }(\% / h) \\
\text { Post-Tl }\end{array}$ \\
\hline Const 2 min & $-39.3 \pm 6.4$ & $-29.8 \pm 4.3$ \\
\hline Const 5 min & $-20.10 \pm 3.1$ & $-16.5 \pm 2.2$ \\
\hline Incr 2-3-5 min & $-15.20 \pm 3.9$ & $-12.8 \pm 3.0$ \\
\hline Const Trues & $-7.7 \pm 5.0$ & $-6.1 \pm 3.9$ \\
\hline
\end{tabular}

BP $_{\text {ND }}$ CURVES SLOPES* RESULTS - HUMAN DATA - PRESTO

\begin{tabular}{|c|c|c|}
\hline Frame Scheme & $\begin{array}{c}\text { Slope } \pm \text { SE }(\% / h) \\
\text { ACC }\end{array}$ & $\begin{array}{c}\text { Slope } \pm \text { SE }(\% / h) \\
\text { Post-Tl }\end{array}$ \\
\hline Const 2 min & $-24.2 \pm 8.2$ & $-22.6 \pm 5.4$ \\
\hline Const 5 min & $-17.2 \pm 7.2$ & $-20.1 \pm 3.6$ \\
\hline Incr 2-3-5 min & $-14.7 \pm 6.1$ & $-17.5 \pm 3.6$ \\
\hline Const Trues & $-7.3 \pm 7.7$ & $-10.2 \pm 4.3$ \\
\hline
\end{tabular}

*Slope - Linear fits from graphs presented in Figure 7 and Figure 10, SE - Standard Error.

\section{Discussion}

In this study, the quantification bias given by different framing schemes was investigated. In addition, an alternative framing scheme (Const Trues) that kept constant true counts per frame in all of the PET dynamics was proposed. The propagation of the bias into the binding was also evaluated by using simple ratio methods at equilibrium. Moreover, the analysis was additionally performed with the PRESTO reconstruction and compared to the vendor's 3D OP-OSEM. Quantification bias as a result of low counts in PET images reconstructed with the 3D OP-OSEM algorithm has been studied by several groups with special emphasis being placed on the impact of the bias on estimated activity concentrations and binding potentials in neuroreceptor studies (8-10, $16,19,26,27)$. At low count rates, the distribution of the reconstructed events per voxel is asymmetric, leading to a bias in the mean of the voxel activity concentration values (9). MLEM 
441 and OP-OSEM reconstructions tend to be biased in regions with low activity concentrations,

442 particularly if these regions are surrounded by regions of high activity concentrations (16).

443 Slambrouck et al., also demonstrated that regions with low activity concentrations will converge

444 much more slowly and a very high number of iterations would be required to eliminate the positive

445 bias (16). However, it is not usual to have a high enough number of iterations to avoid this

446 convergence bias. Moreover, since there are other sources for bias apart from iteration numbers, a

447 completely bias-free image is not possible (16) and at low count statistical levels, other factors,

448 such as random estimation, can be critical. This is because single rates are not constant and/or the

449 activity distribution is not static during the frame (at this point framing schemes should be carefully

450 chosen). Previous works have reduced the random events estimation bias considerably by applying

451 a reduction variance given by VRR algorithms $(9,14,19)$. However, other factors, such as scatter

452 overcorrection, global dead time correction instead of block-wise correction, inconsistences in the

453 attenuation map, etc., can still contribute to the reconstruction bias. These factors are not within

454 the scope of this work.

Other methods to reduce bias at low count rates have been proposed, e.g. Hong et al. (28)

used a method called complementary frame reconstruction. This method involves the indirect

457 formation of a low count image (short time frame) through the subtraction of two frames with 458 longer acquisition times (high statistics). The short time frame is then excluded from the second, 459 long frame data prior to reconstruction. The method was tested with a phantom and with clinical 460 data using HRRT and Biograph mCT scanners and with OP-OSEM reconstruction. In contrast to 461 this study, the authors focused their work on applications relating to estimation of the arterial input 462 function, although they also commented on potential future applications. While, our alternative 463 framing scheme, (Const Trues), is intended to mitigate the bias in any group of reconstructed 
464 images, so far it was validated for equilibrium methods at low count rates and particularly for BI 465 protocols when used together with ABP and simultaneously MR sequences. The goal here is to 466 maximize the possibility to identify pre and post task effects together with other modalities and 467 during the same brain conditions. In addition, it increases the possibility to detect binding 468 differences by reducing the bias and keeping at the same levels during the pre-challenge and post469 challenge time periods for a possible comparison. Since the bias cannot be avoided entirely, even 470 when using 3D OP-OSEM + VRR reconstructions, we hypothesize that, if the bias is mainly due 471 to the low count rates, the bias can be mitigated and maintained at a constant level by keeping the 472 counts per frame constant during all PET dynamics (keeping bias constant for the all acquisition 473 time interval).

The findings of our study support a previous bias investigation undertaken by Planeta475 Wilson and colleagues using HRRT and OP-OSEM (MOLAR) reconstruction. Here, a bias of $476-4 \pm 2 \%$ was shown for GM (high activity concentration region) and $4 \pm 5 \%$ for white matter (low 477 activity concentration) regions (7). In terms of bias range, for low and high activity concentration 478 regions, our results showed agreement, since the bias range values were closer for BG (with $\pm 3 \%$ ) 479 and $\mathrm{Hot}_{1}$ (between $-3 \%$ and $5 \%$ ) with respect to activity concentration accuracy. In addition, based 480 on data obtained from a ${ }^{11} \mathrm{C}$ filled decay phantom study and a human data (slopes), we also 481 observed a negative bias for high activity concentration regions when the frame length was 482 shortened (low counts). Our study differs from previous studies reported in the analysis approach 483 since we additionally evaluated the slope changes from a complete dynamic PET acquisition rather 484 than just a single frame with different count statistics. Furthermore, our results completely 485 diverged from others in terms of the amount of bias found. Johansson et al., reported a significantly 486 higher bias which was in the range of $-16 \%$ to $-18 \%$ in high activity concentration regions $(1 \mathrm{M}$ 
487 and 200k counts, respectively) using HRRT and 3D OP-OSEM. van Velden et al., reported a bias 488 of around $-9 \%$ to $-14 \%$ in GM (Hoffman phantom in 5 seconds frame) using the same scanner 489 and reconstruction $(5,19)$. Moreover, Reilhac and colleagues reported a bias of up to $80 \%$ in the 490 CER region for the end of the activity time course in simulations using 3D UW-OSEM (8).

491 It is important to interpreter these results carefully since our study differs in some aspects, for 492 example, in terms of the activity concentration and high to low activity concentration ratios used, 493 the radioisotope and radiopharmaceuticals, and the range of analyzed statistics. Moreover, there 494 are some configuration differences between scanners and the 3D OSEM reconstruction procedures 495 presented (number of iterations and subsets), post-processing smoothing, etc. However, in the 496 interest of comparability, we tried to compare our results with the closest 3D OP-OSEM 497 procedures and statistics as possible.

In terms of bias propagation into binding values, our study is in agreement with van Velden et al (19), where a bias of - $14 \% \mathrm{BP}_{\mathrm{ND}}$ was reported when using reference tissue models. In our 500 study, a $\mathrm{BP}_{\mathrm{ND}}$ bias of up to $-40 \% \pm 6 \%$ per hour (Const $2 \mathrm{~min}$ ) was found and when mitigated 501 with the Const Trues framing scheme, the bias was $-7 \% \pm 5 \%$ per hour in ACC (observed as slopes 502 in the human data example, see in Table 6). It is important to emphasize that our approach is based 503 on $\mathrm{BI}$ studies and the $\mathrm{BP}_{\mathrm{ND}}$ values in other studies were obtained from bolus only analysis applying 504 kinetic modeling instead of simple ratio methods. We hypothesize, that pure bolus and pure 505 infusion studies may suffer from bias issues similar to those found in our study and that it might 506 be possible to mitigate the bias with the proposed Const Trues framing approach. As the bias is 507 introduced during iterative image reconstruction, parameters estimated via kinetic modeling will 508 potentially be affected as well. However, further studies for evaluating this are needed. 
The slopes approach was used in this study because we expected a more similar bias

510

511

512

513

514

515

516

517

518

519

520

521

522

523

524

525

526

527 release and are thus expected to be smaller than pharmacological tasks (e.g. video game playing

528 tasks with $\left[{ }^{11} \mathrm{C}\right]$ raclopride, $13 \%$ BP decrease versus psychostimulants $10 \%$ to $20 \%$ BP decrease

529 (29)). In such a case, the bias could mask the effect in the Hot H $_{1}$ region, since it would be larger than

$53010 \%$ during the time interval T4 and 5\% during the time interval T6, when a scheme with Const

between time intervals (T4, T5 and T6) and along the TACs when applying the Const Trues

framing scheme. In this case, the slope should not be changed by the bias. Nevertheless, bias

between subjects can. We noticed the same trend as found in the ${ }^{11} \mathrm{C}$ filled phantom decay study, where the bias became negative along the TAC, especially in time interval T6. Furthermore, it was also possible to identify a stronger bias in a smaller region with high activity concentration, represented by $\mathrm{Hot}_{2}$ (simulating nucleus accumbens volume). Unfortunately, this region showed consistently larger bias values, especially for BP. The negative bias for activity concentration was in the $1 \%$ to $-8 \%$ range, and the variability was higher between the different time intervals (T4, T5 and T6) leading to larger BP mean bias values of up to - 10\%. The high BP bias values and its high variability for regions comparable to $\mathrm{Hot}_{2}$ in size could cause spurious results during neuroreceptor study analysis. Walker and colleagues (9) found $-13 \%$ without VRR and $-5.5 \%$ with VRR bias (for 1.7M counts) in the caudate head $(1.3 \mathrm{ml})$ which is of similar size to $\mathrm{Hot}_{2}(1.13 \mathrm{ml})$.

It should also be noted, that the negative trend of bias in hot regions and the positive trend of bias in the BG region leads to an amplified underestimation of BP (Equation 3). This is an important point of consideration for neuroreceptor studies using equilibrium conditions, especially if the aim of the study is to evaluate the binding prior, during, and post a specific task. This holds especially for cognitive tasks where the potentially induced effects are caused by endogenous frames is used (see in Figure 4). This effect can also be observed for ABP in-vivo TACs (see 
532 Figure 6, Figure 7 and Table 3). Here, we observed a relative change of up to - $40 \%$ per hour in 533 the ACC region with the vendor's 3D OP-OSEM reconstruction and with the Const 2 min framing 534 scheme. This could lead to misinterpretation of the effects caused by the task and give rise to 535 incorrect conclusions. With the proposed, alternative Const Trues framing scheme, the average BP 536 bias could be reduced/mitigated to mean values around 5\% in the phantom study (see Figure 8) 537 which is constant for all intervals T4, T5, and T6. A drawback of the proposed framing method is 538 the use of the very short time frames at the beginning of the acquisition, which result from the 539 reference counts number in the last frame. This also leads to an increased variability (Figure 8). 540 Nevertheless, the time required to reach equilibrium in neuroreceptor studies, e.g. for ABP usually 541 starts at around 30 minutes p.i., so this drawback is tolerable for our studies, since the relevant part 542 of the PET study is during the equilibrium phase interval (see Table 6). Note that the SE values 543 remain constant with our Const Trues framing scheme (Figure 7 and Figure 10).

544 For the Incr 2-3-5 min framing, a reduction in the BP mean bias values was also observed. 545 However, regarding the difference between time intervals T4, T5 and T6, a higher bias (around $54610 \%$ ) can be seen in T4 for Incr schemes compared to Const Trues (around 5\% from T4 to T6).

547 From these findings, framing schemes with constant frame lengths are less suitable for the 548 evaluation of $\mathrm{BP}_{\mathrm{ND}}$ when is required for the analysis prior and post effects in neuroreceptor studies 549 as the bias range has high variability between the time intervals (from $\mathrm{T} 4=15 \%$ to $\mathrm{T} 6=5 \%$ ). In

550 Figure 5, the falling and rising trends of the bias for the BG region and the falling behavior of the 551 bias for the hot regions can be clearly observed for all studied framing schemes. The similar results 552 were found for the TACs in the human brain study with $\left[{ }^{11} \mathrm{C}\right] \mathrm{ABP} 688$ (especially the rising 553 behavior in the last time interval for CER and falling behavior for ACC, see Figure 6). This finding 
554 was also noticed by van Velden et. al. (19) using reference region (pons) approaches with $555 \quad\left[{ }^{11} \mathrm{C}\right]$ Flumazenil.

556 When comparing different reconstructions procedures, PRESTO showed slightly lower bias values 557 for regions with low activity concentration (BG and CER) and rising trends, which were smaller, 558 compared to the vendor's 3D OP-OSEM (especially for Const frame schemes). Furthermore, when 559 using PRESTO for the phantom study, lower slope values (relative change per hour) were noticed 560 in the time interval T6 for the BG VOI compared to the vendor's 3D OP-OSEM reconstruction. 561 However, when evaluating the accuracy of the BP values for both reconstructions, the remaining, 562 optimized bias was around 5\% for the vendor's 3D OP-OSEM compared to around $10 \%$ for 563 PRESTO with the Const Trues. We attribute this difference to the crosscalibration between 564 PRESTO and the vendor's 3D OP-OSEM reconstruction. The PRESTO reconstruction lead to a 565 slightly smaller bias for low activity concentration regions during the final time interval (T6) in 566 the ${ }^{11} \mathrm{C}$ filled phantom decay study. For the ABP TACs, a smaller range of the relative change per 567 hour and bias values closer to zero were observed with PRESTO in the low activity concentration 568 regions when compared to the vendor's 3D OP-OSEM reconstruction for all tested framing 569 schemes.

570 In summary, all framing schemes methods, even with the minimized bias range due to our 571 proposed framing scheme Const Trues, produce a bias of at least 5\%, which should be taken into 572 account for the conclusions drawn from the observed $\mathrm{BP}_{\mathrm{ND}}$ values. Moreover, when task effects 573 are evaluated with the mitigated bias framing scheme (Const Trues), the task must induce a change 574 sufficiently larger than $5 \%$ in order to be observable in equilibrium studies with ABP during the 575 analyzed time interval. 
576 Further studies are planned to investigate the influence of scatter correction at low count rates, as

577 it has already been pointed out by Jian and colleagues (10) that scatter correction is a potential bias

578 source.

$579 \quad$ Conclusion

580 This work aimed to analyze the bias in activity concentrations estimated from PET images and

581 its propagation into binding potential values for equilibrium studies using a ${ }^{11} \mathrm{C}$ filled decay

582 phantom study and a human data set from a BI study with ABP. Taking into account all framing

583 schemes tested, one can conclude that PRESTO showed lower bias values for the low activity

584 concentration region, particularly when using the Const Trues framing scheme method proposed.

585 Therefore, in order to be observable, the size of any potentially endogenous response to tasks or

586 challenges in neuroreceptor studies with ABP using the BI protocol should be sufficiently larger

587 than 5\% for our proposed framing scheme and when using vendor's reconstruction, since the bias

588 propagates into the biding potential values. Further studies are required to estimate the bias 589 introduced by other sources, such as scatter correction.

590

591 Declaration

592

593 Ethics Approval and Consent to Participate

594 Our work has been carried out in accordance to The Code of Ethics of the World Medical 595 Association (Declaration of Helsinki) for experiments. All participants (human example) signed 596 written informed consents in addition to verbal consent. The privacy rights of human subjects were 597 observed. The ICMJE Recommendations for the Conduct, Reporting, Editing, and Publication of 598 Scholarly Work in Medical Journals have been followed. 
599

600

601

602

603

604

605

606

607

608

609

610

611

612

613

614

615

616 617 PET study design discussions, revision and corrections of the manuscript. ERK: MR-PET

618

619

\section{Consent for Publication}

All authors had full access to all data in the study. We confirm that the manuscript has been read and approved for submission by all the named co-authors.

\section{Availability of data and materials}

The datasets used and analyzed during the current study are available from the corresponding author on reasonable request.

\section{Disclosure/Competing Interests}

These data are part of the Dr. rer. medic. thesis at the Medical Faculty of the RWTH Aachen University, Germany of Cláudia Régio Brambilla. No potential conflict of interest relevant to this article was reported.

\section{Funding}

This work was supported in part by the EU-FP7 funded project TRIMAGE (Nr. 602621), and Cláudia Régio Brambilla thanks to DAAD for financial support (Nr. 57299294).

\section{Author contributions}

CRB: PET bolus-infusion protocol optimization, data acquisition, PET imaging data analysis, statistical design and analysis, manuscript writing, correction and revision. JS: PET reconstruction discussions, PRESTO reconstruction, manuscript revision and correction. AI: script for PET True events curve extraction and manuscript revision. $L T$ : MR-PET hardware and data acquisition. $H H$ : attenuation correction and revision of the manuscript. NJS: MR-PET hardware and revision of the manuscript. IN: ABP study design and setup, approval ethics and BfS and revision of the 
manuscript. $C L$ : study design and setup, data analysis revision, manuscript corrections and

revision.

\section{Acknowledgment}

\section{References}

1. Shepp LA, Vardi Y. Maximum likelihood reconstruction for emission tomography. IEEE Trans Med Imaging. $1982 ; 1(2): 113-22$. Hudson HM, Larkin RS. Accelerated image reconstruction using ordered subsets of projection data. IEEE Trans Med Imaging. 1994;13(4):601-9.

3. Barrett HH, White T, Parra LC. List-mode likelihood. J Opt Soc Am A Opt Image Sci Vis. 1997;14(11):2914-23.

4. Liu X, Comtat C, Michel C, Kinahan P, Defrise M, Townsend D. Comparison of 3-D reconstruction with 3D-OSEM and with FORE plus OSEM for PET. leee T Med Imaging. 2001;20(8):804-14.

6385 5. Johansson J, Clikonen V, Teraes M. Quantitative brain imaging using the new, fast iterative histogram-mode reconstruction for the HRRT PET scanner. leee Nucl Sci Conf R. 2007:3463-7.

640 6. van Velden FH, Kloet RW, van Berckel BN, Lammertsma AA, Boellaard R. Accuracy of 3-dimensional reconstruction algorithms for the 641 high-resolution research tomograph. J Nucl Med. 2009;50(1):72-80.

642 7. Planeta-Wilson B, Yan J, Mulnix T, Carson RE. Quantitative Accuracy of HRRT List-mode Reconstructions: Effect of Low Statistics. IEEE 643 Nucl Sci Symp Conf Rec (1997). 2008;2008:5121-4.

$644 \quad 8 . \quad$ Reilhac A, Tomei S, Buvat I, Michel C, Keheren F, Costes N. Simulation-based evaluation of OSEM iterative reconstruction methods in 645 dynamic brain PET studies. Neuroimage. 2008;39(1):359-68.

646 9. Walker MD, Asselin MC, Julyan PJ, Feldmann M, Talbot PS, Jones T, et al. Bias in iterative reconstruction of low-statistics PET data: 647 benefits of a resolution model. Phys Med Biol. 2011;56(4):931-49.

648 10. Jian Y, Planeta B, Carson RE. Evaluation of bias and variance in low-count OSEM list mode reconstruction. Phys Med Biol. 2015;60(1):15-

12. Cloquet C, Defrise M. MLEM and OSEM Deviate From the Cramer-Rao Bound at Low Counts. IEEE Transactions on Nuclear Science. 2013;60(1):134-43.

13. Chen TB, Lu HHS, Kim HK, Son YD, Cho ZH. Accurate 3D reconstruction by a new PDS-OSEM algorithm for HRRT. Radiat Phys Chem. 2014;96:107-14.

14. Byars LG, Sibomana M, Burbar Z, Jones J, Panin V, Barker WC, et al. Variance reduction on randoms from delayed coincidence histograms for the HRRT. 2005 leee Nuclear Science Symposium Conference Record, Vols 1-5. 2005:2622-6.

15. Watson CC. New, faster, image-based scatter correction for 3D PET. leee T Nucl Sci. 2000;47(4):1587-94.

16. Van Slambrouck K, Stute S, Comtat C, Sibomana M, van Velden FH, Boellaard R, et al. Bias reduction for low-statistics PET: maximum likelihood reconstruction with a modified Poisson distribution. IEEE Trans Med Imaging. 2015;34(1):126-36. 
18. Herzog H, Langen KJ, Weirich C, Rota Kops E, Kaffanke J, Tellmann L, et al. High resolution BrainPET combined with simultaneous MRI. Nuklearmedizin. 2011;50(2):74-82.

19. van Velden FH, Kloet RW, van Berckel BN, Wolfensberger SP, Lammertsma AA, Boellaard R. Comparison of 3D-OP-OSEM and 3D-FBP reconstruction algorithms for High-Resolution Research Tomograph studies: effects of randoms estimation methods. Phys Med Biol. 2008;53(12):3217-30.

20. Association NEM. NEMA Standards Publication NU 2-2007: Performance Measurements of Positron Emission Tomographs: National Electrical Manufacturers Association; 2007.

21. McCollum LA, Walker CK, Roche JK, Roberts RC. Elevated Excitatory Input to the Nucleus Accumbens in Schizophrenia: A Postmort em Ultrastructural Study. Schizophrenia Bulletin. 2015;41(5):1123-32.

22. Regio Brambilla C, Veselinovic T, Rajkumar R, Mauler J, Orth L, Ruch A, et al. mGluR5 receptor availability is associated with lower levels of negative symptoms and better cognition in male patients with chronic schizophrenia. Hum Brain Mapp. 2020.

23. Elmenhorst D, Mertens K, Kroll T, Oskamp A, Ermert J, Elmenhorst EM, et al. Circadian variation of metabotropic glutamate receptor 5 availability in the rat brain. J Sleep Res. 2016;25(6):754-61.

24. Kops ER, Herzog H, Shah NJ. Comparison template-based with CT-based attenuation correction for hybrid MR/PET scanners. EJNMMI Phys. 2014;1(Suppl 1):A47.

25. Ametamey SM, Treyer V, Streffer J, Wyss MT, Schmidt M, Blagoev M, et al. Human PET studies of metabotropic glutamate receptor subtype 5 with 11C-ABP688. J Nucl Med. 2007;48(2):247-52.

26. Belanger MJ, Mann JJ, Parsey RV. OS-EM and FBP reconstructions at low count rates: effect on 3D PET studies of [C-11] WAY-100635. Neuroimage. 2004;21(1):244-50.

27. Haggstrom I, Axelsson J, Schmidtlein CR, Karlsson M, Garpebring A, Johansson L, et al. A Monte Carlo study of the dependence of early frame sampling on uncertainty and bias in pharmacokinetic parameters from dynamic PET. J Nucl Med Technol. 2015;43(1):53-60.

28. Hong I, Cho SH, Michel CJ, Casey ME, Schaefferkoetter JD. Complementary frame reconstruction: a low-biased dynamic PET technique for low count density data in projection space. Physics in Medicine and Biology. 2014;59(18):5441-55.

29. Laruelle M. Imaging synaptic neurotransmission with in vivo binding competition techniques: a critical review. J Cereb Blood Flow Metab. 2000;20(3):423-51.

\title{
List of Abbreviations
}

$\left[{ }^{11} \mathrm{C}\right] \mathrm{ABP} 688$ - (3-(6-methyl-pyridine-2-ylethynyl)-cyclohex-2-enone-O-[11C]-methyloxime)

\section{D OP-OSEM - 3D Ordinary Poisson Ordered Subset Expectation Maximization}

\author{
ACC - Anterior Cingulate Cortex
}

$$
\mathrm{BG} \text { - background region }
$$

BI - Bolus plus Infusion

BP - Binding Potential - related to phantom data in this work

$$
\mathrm{BP}_{\mathrm{ND}} \text { - non-displaceable Binding Potential - related to human data in this work }
$$

CER - Cerebellum

EEG - electroencephalography

GM - grey matter cortex

LOR - Line of Response

mGluR5 - metabotropic glutamatergic receptor type 5

ML-EM - Maximum Likelihood - Expectation Maximization

MR - Magnetic resonance 
704 PET - Positron Emission Tomography

705 Post_Tl - Posterior Temporal Cortex

706 PVE - Partial Volume Effect

707 SE - Standard Error

708 SSS - Single Scatter Simulation

709 TAC - Time Activity Curve

710 VOI - volume of interest

711 VRR - Variance Reduction Algorithm 


\section{Figures}

a)

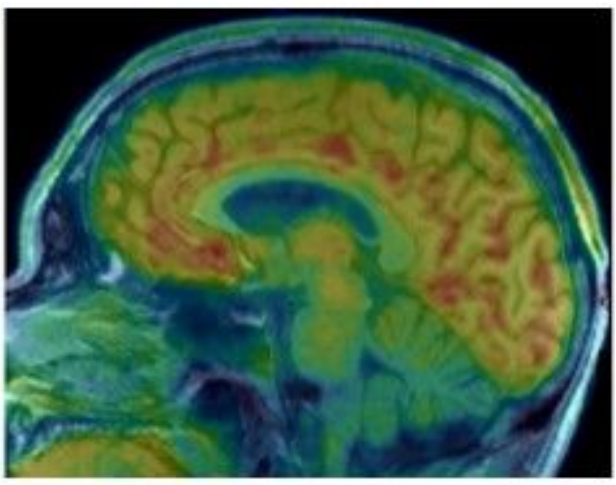

b)

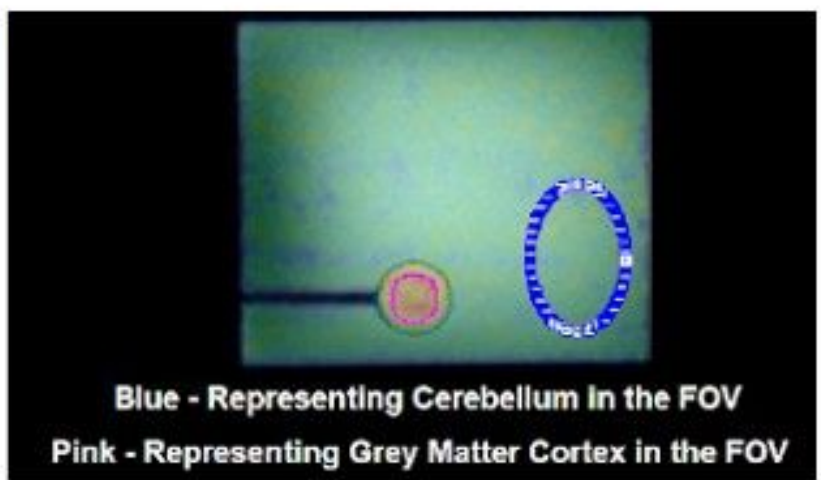

Figure 1

a) Sagittal PET image from the ABP study overlaid with MR T1 image and b) Sagittal PET image of the NEMA phantom overlaid with an MR T1 image during the 11C filled phantom decay study. The high activity concentration region (pink VOI) and low activity concentration region (blue $\mathrm{VOI}$ ) is highlighted for bias analysis.
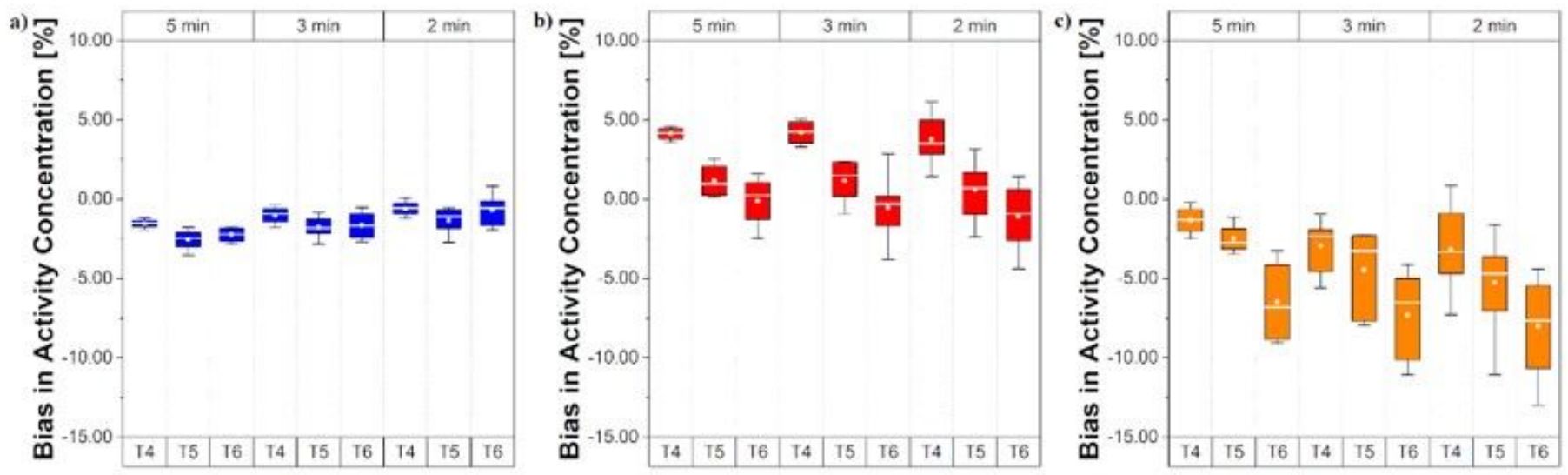

\section{Figure 2}

Bias and variability in activity concentration for a) BG region (low activity concentration), b) Hot1 region (high activity concentration) and c) Hot2 region (high activity concentration); all with vendor's reconstruction. 

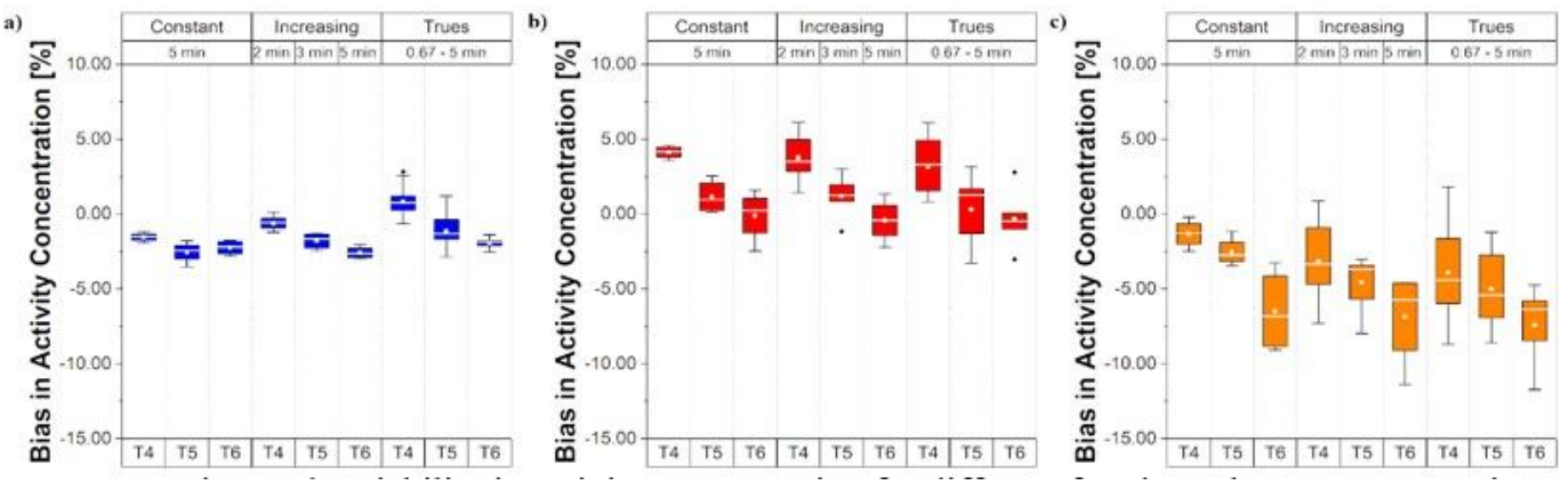

\section{Figure 3}

Bias and variability in activity concentration for different framing schemes a) BG region (low activity concentration), b) Hot1 region (high activity concentration) and c) Hot2 region (high activity concentration); all with vendor's 3D OP-OSEM reconstruction.

a)

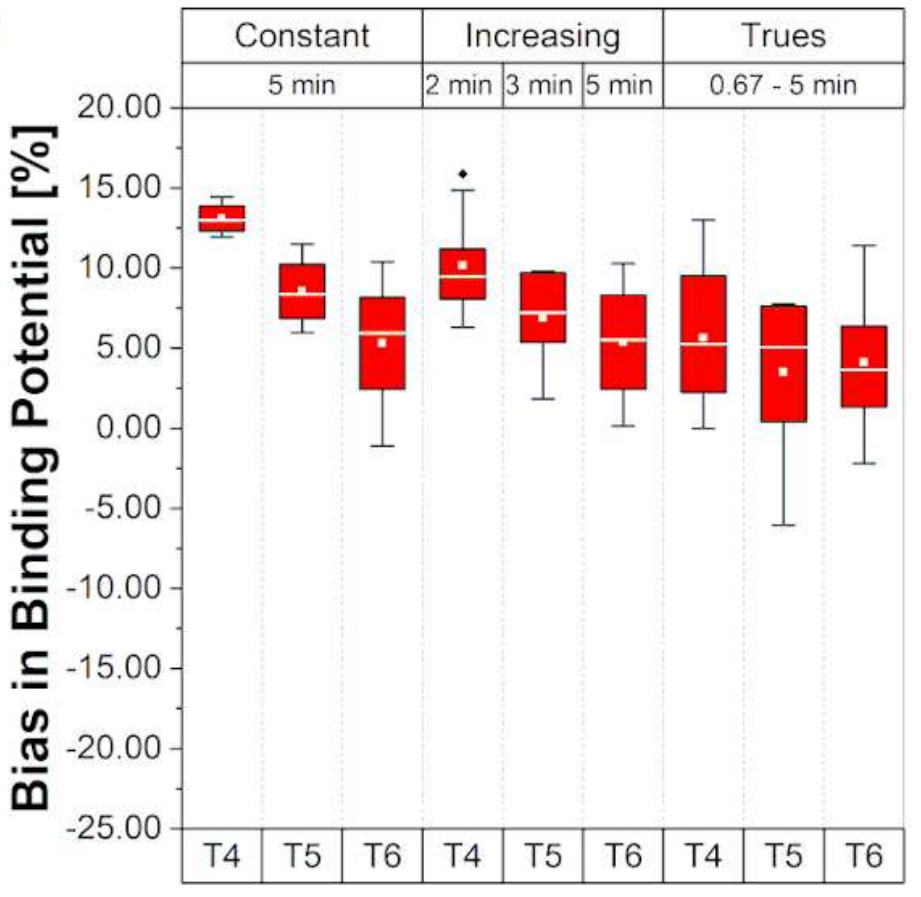

b)

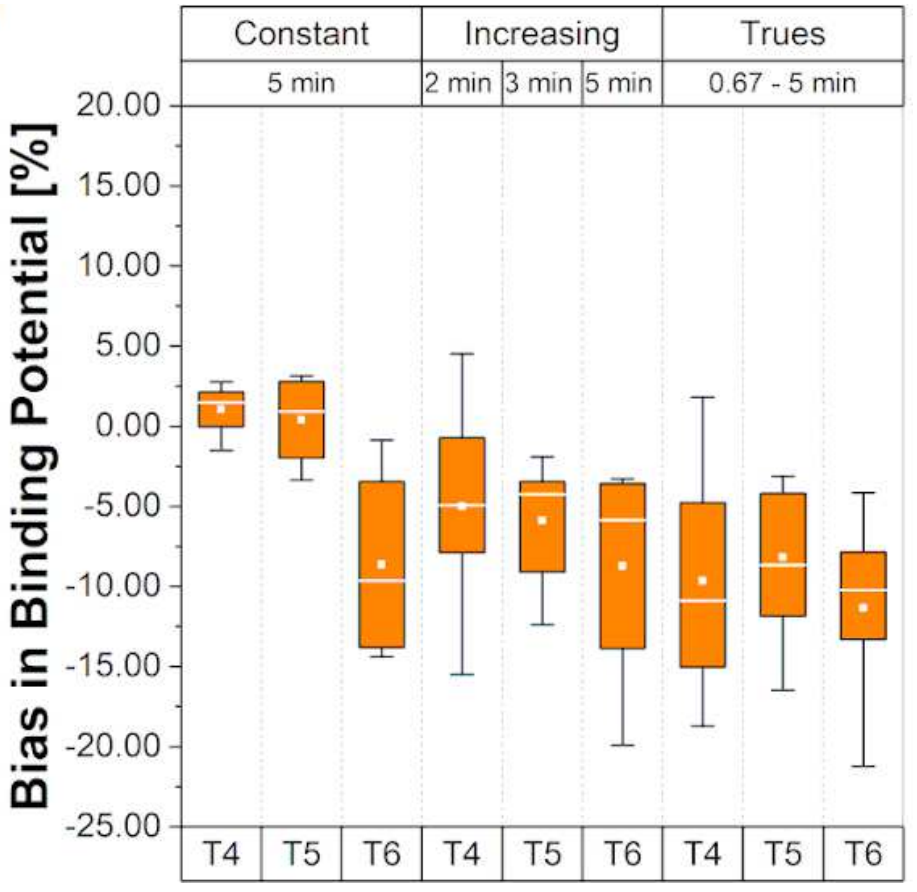

Figure 4

Bias and variability of BP values for a) Hot1 region, and b) Hot2 region; all with vendor's 3D OP-OSEM reconstruction. 
a)
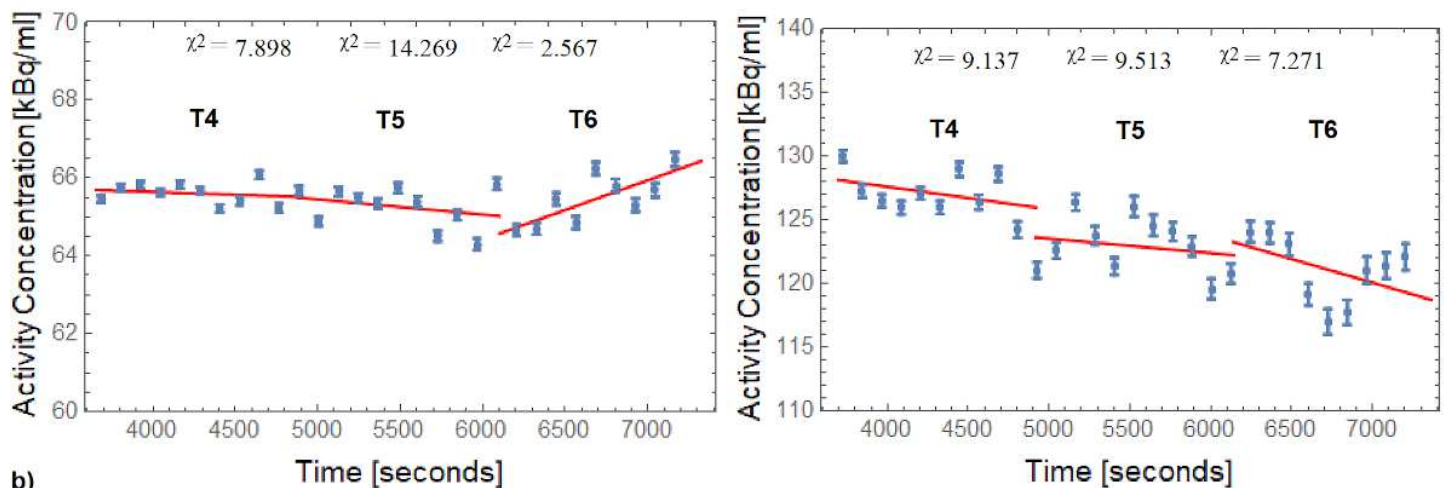

b)
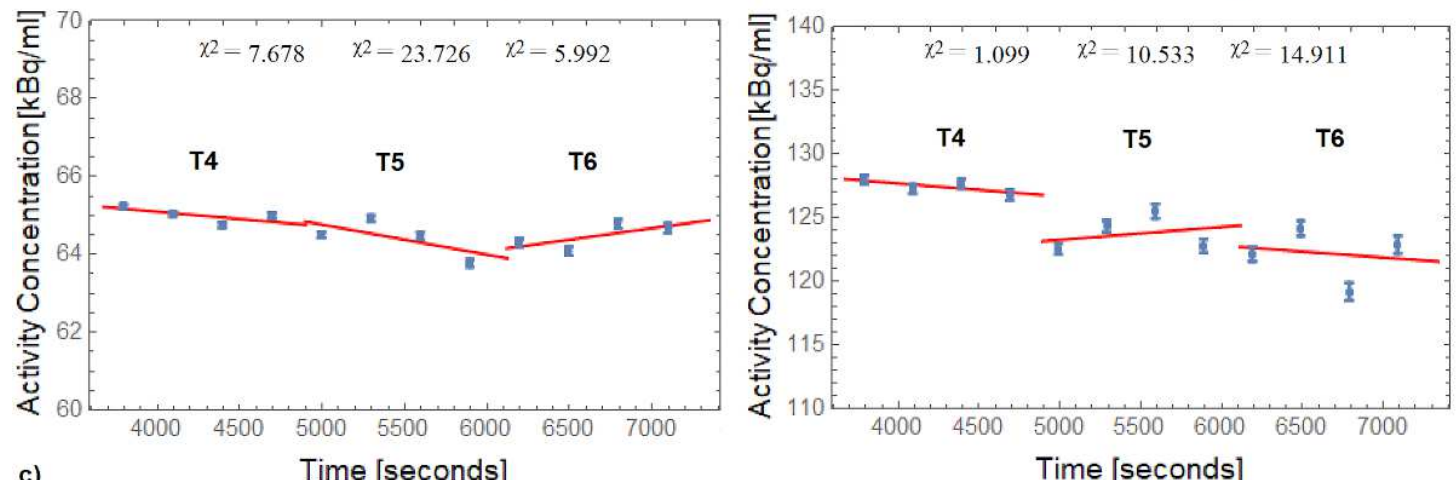

c)

Time [seconds]

Time [seconds]
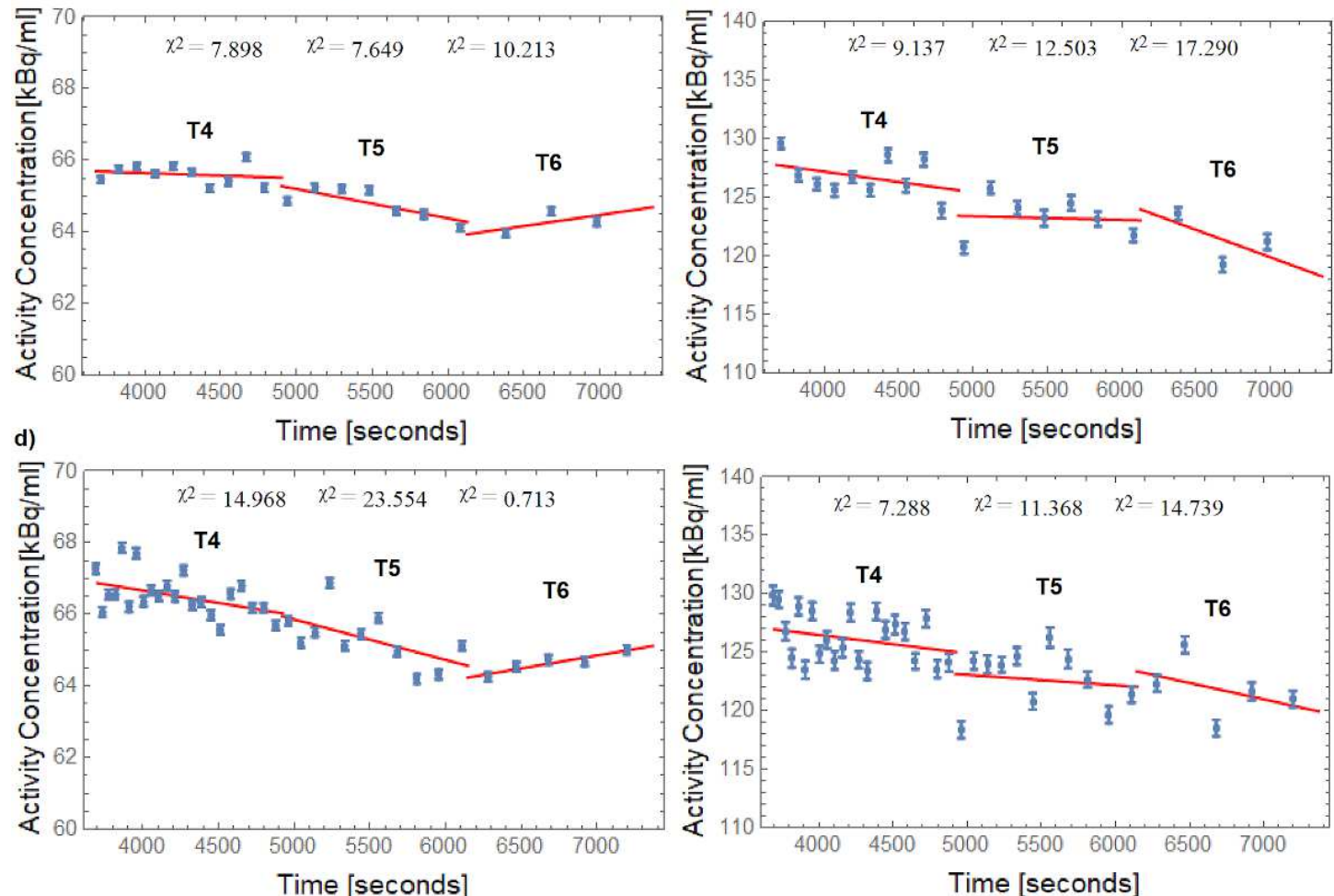

Time [seconds]

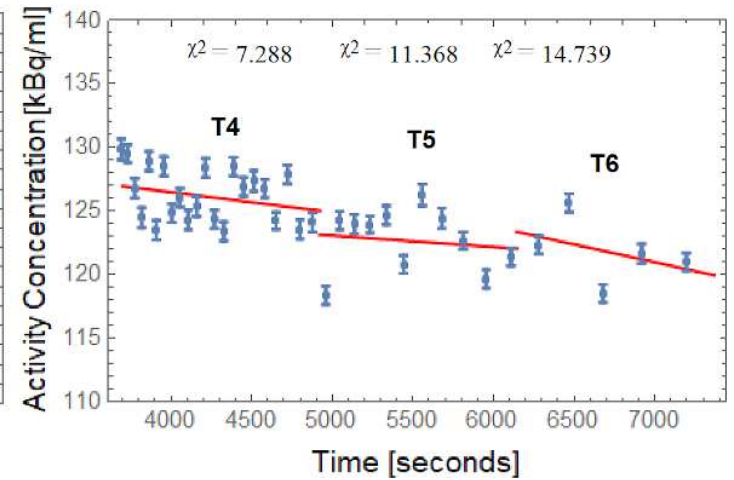

\section{Figure 5}

TACs and linear fits for BG (left side) and Hot1 (right side) regions for a) Const 2min, b) Const 5min, c) Incr 2-3-5 min and d) Const Trues; all with vendor's 3D OP-OSEM reconstruction. $\mathbf{2}^{2}$ : goodness of linear fit. 

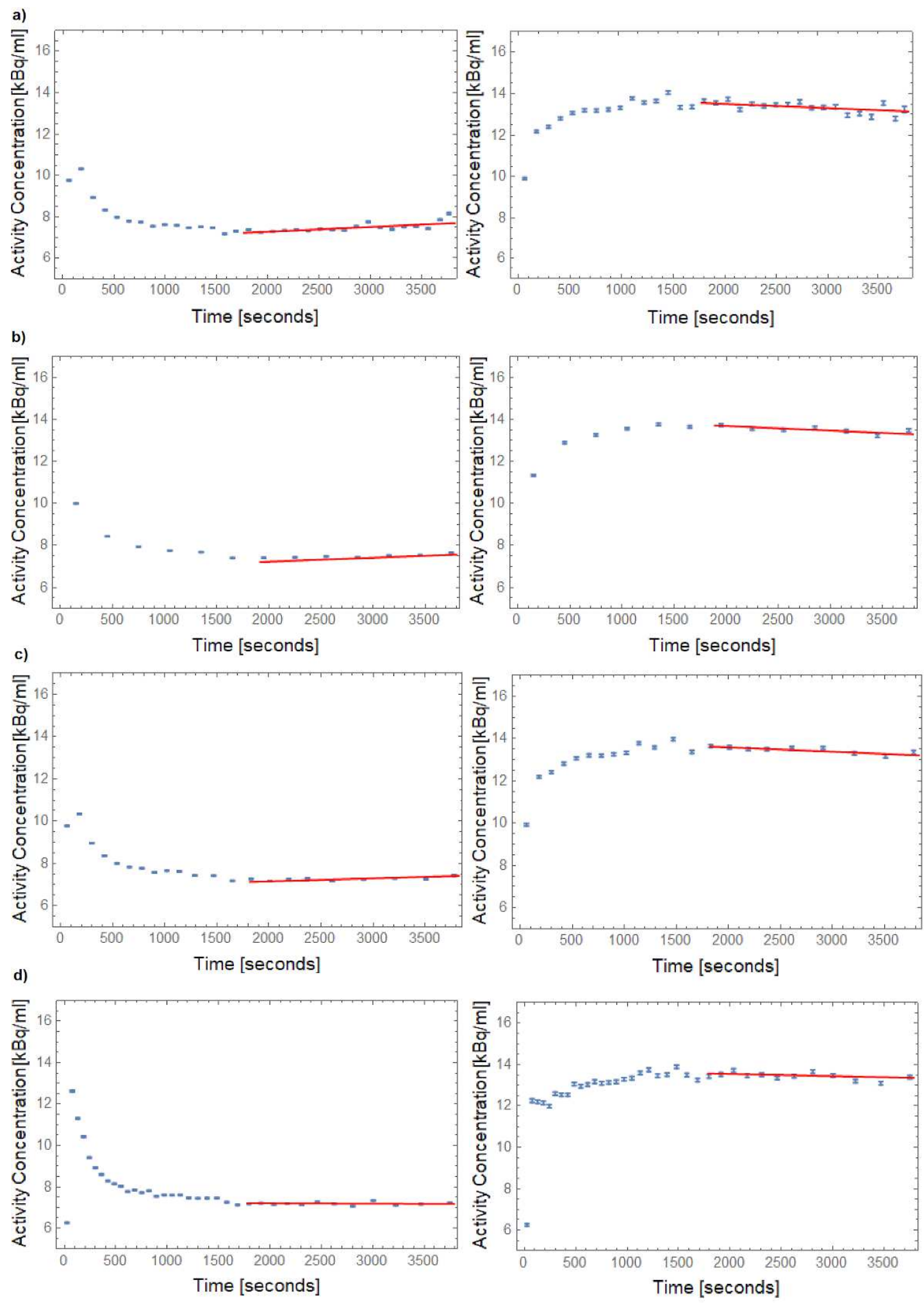

\section{Figure 6}

TACs and linear fits during equilibrium in CER (left side) and ACC (right side) regions from a human brain study for a) Const 2 min, b) Const 5 min, c) Incr 2-3-5 min and d) Const Trues; all with vendor's 3D OPOSEM reconstruction. 
a)
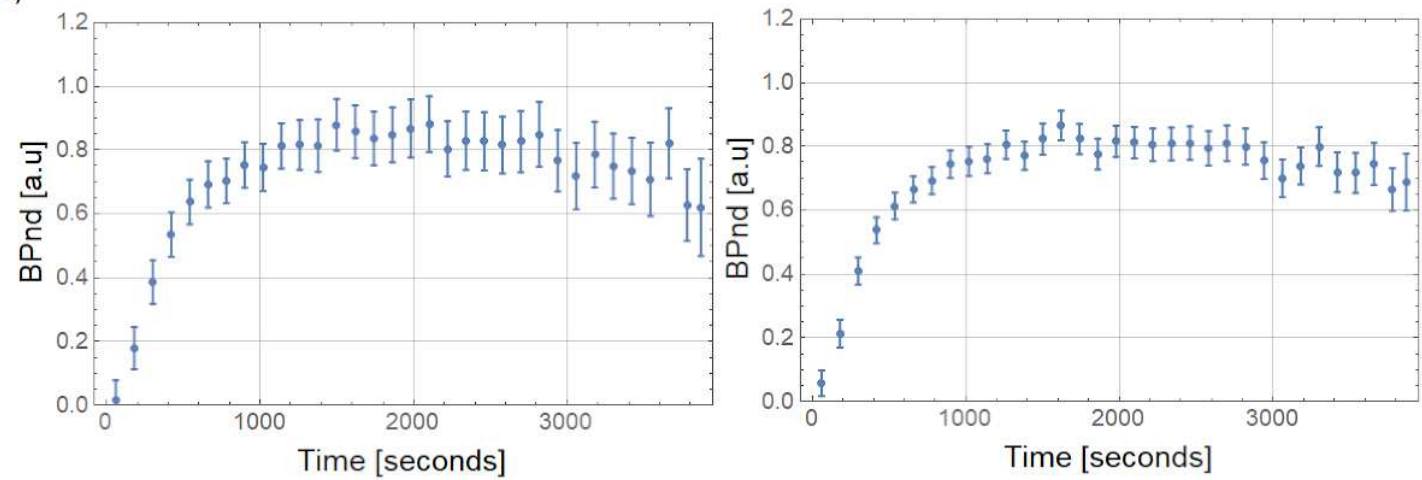

b)
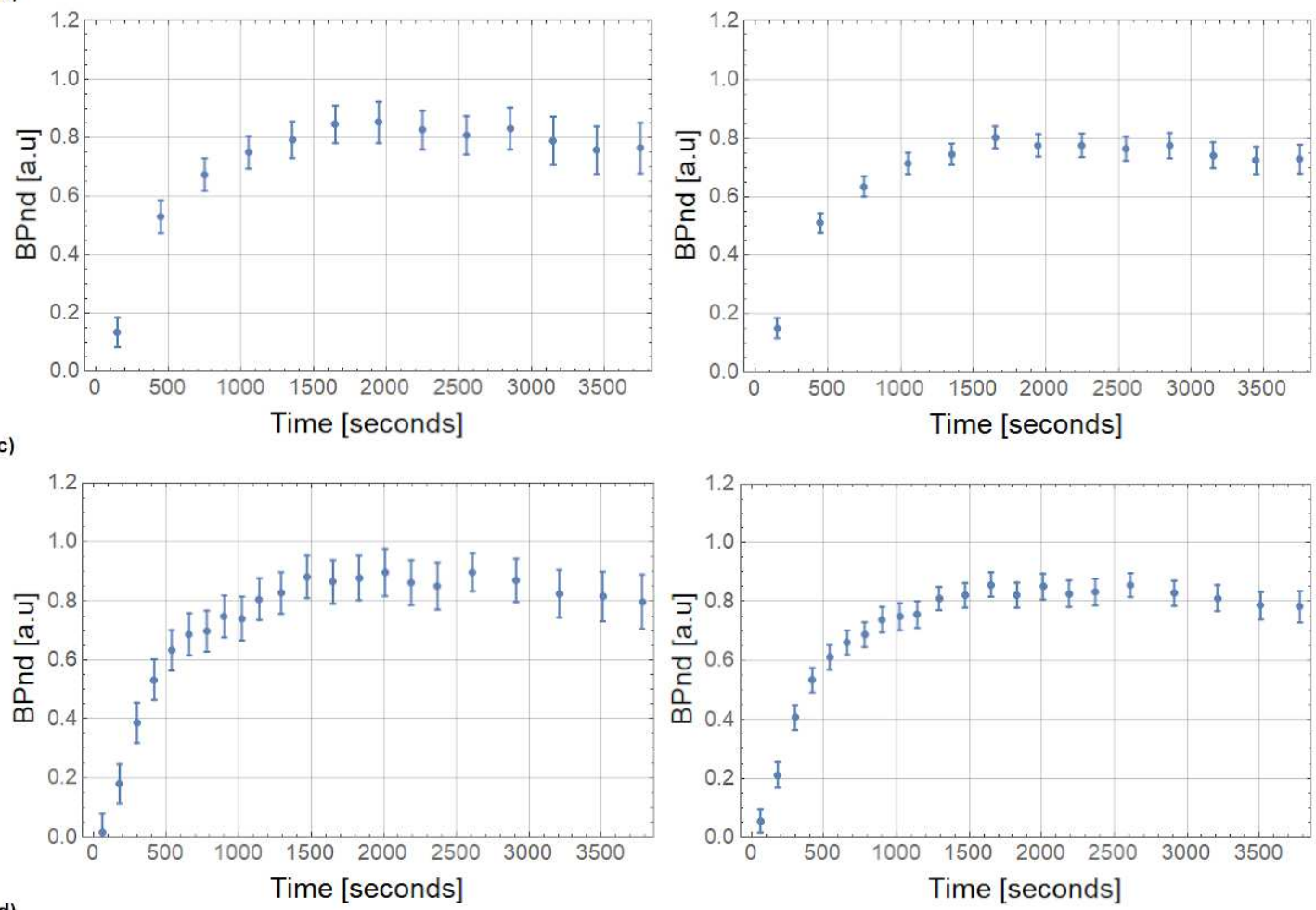

d)
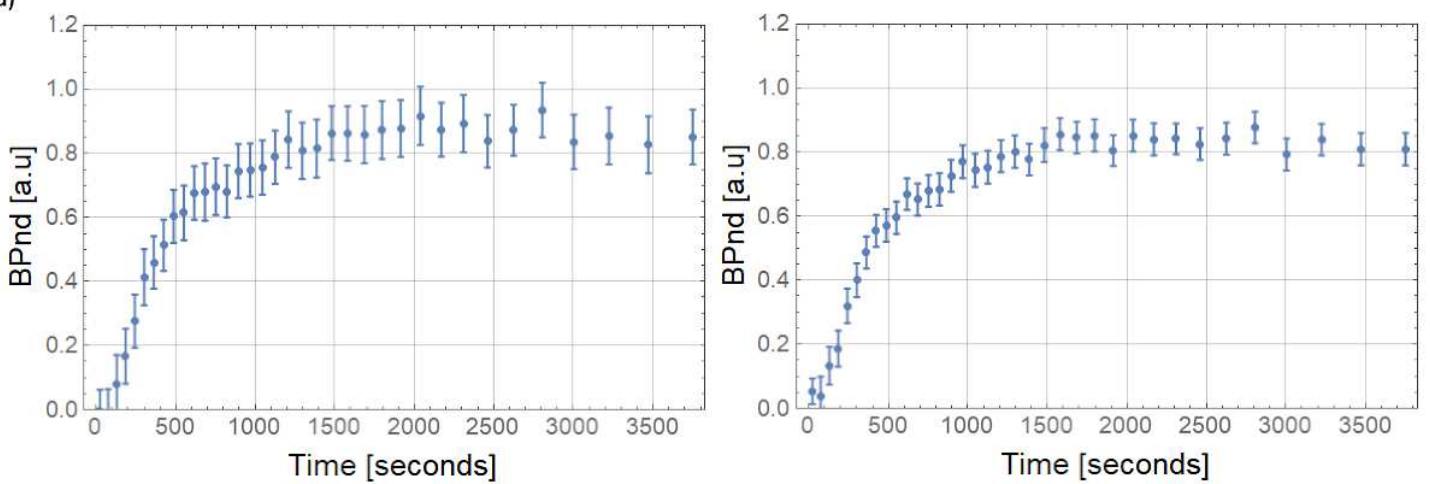

\section{Figure 7}

Values of BPND \pm SE for ACC (left side) and Post-TI (right side) in a human brain study for a) Const 2 min, b) Const 5 min, c) Incr 2-3- 5 min and d) Const Trues; all with vendor's 3D OP-OSEM reconstruction. 

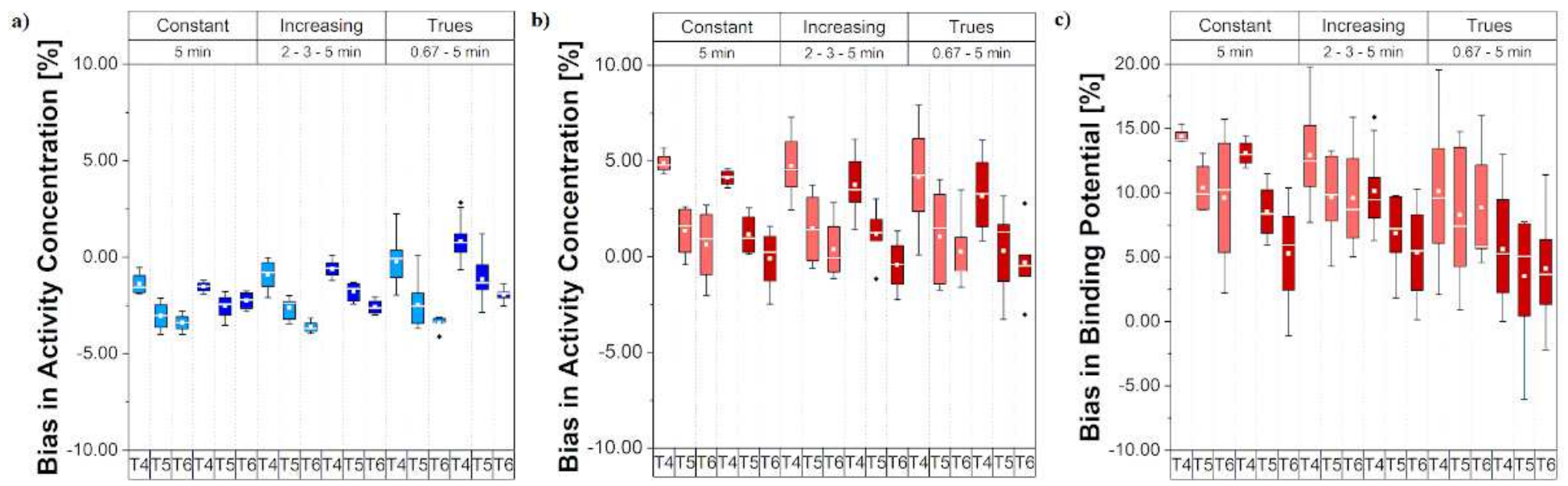

Figure 8

Bias in activity concentrations and BP values for different framing schemes comparing PRESTO (light blue, light red) and vendor's 3D OP-OSEM (dark blue, dark red) in a) BG, b) Hot1 and c) Hot1 BP. 

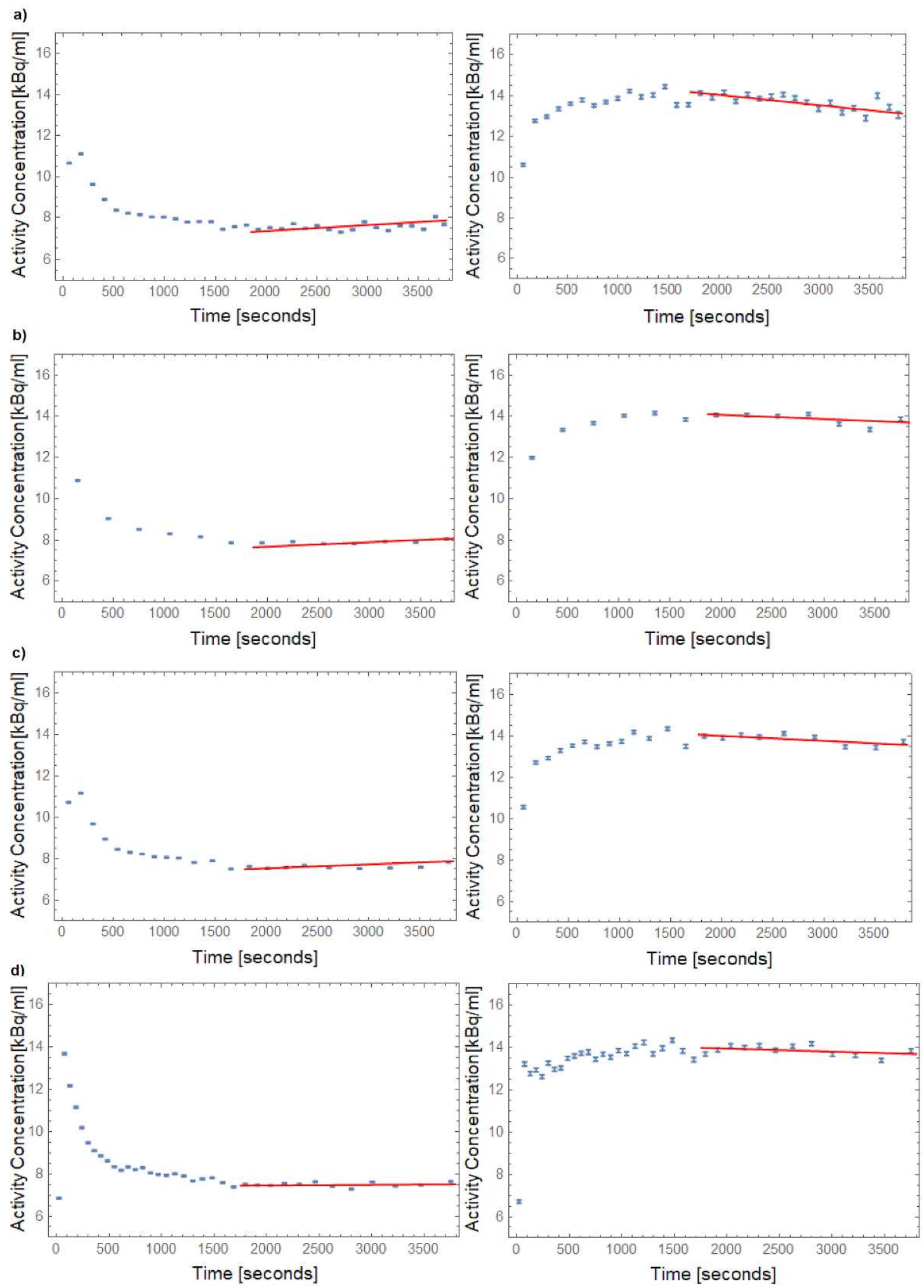

Figure 9

TACs and linear fits during equilibrium in CER (left side) and ACC (right side) regions from a human brain study for a) Const 2 min, b) Const 5 min, c) Incr 2-3-5 min and d) Const Trues; all with PRESTO reconstruction. 
a)
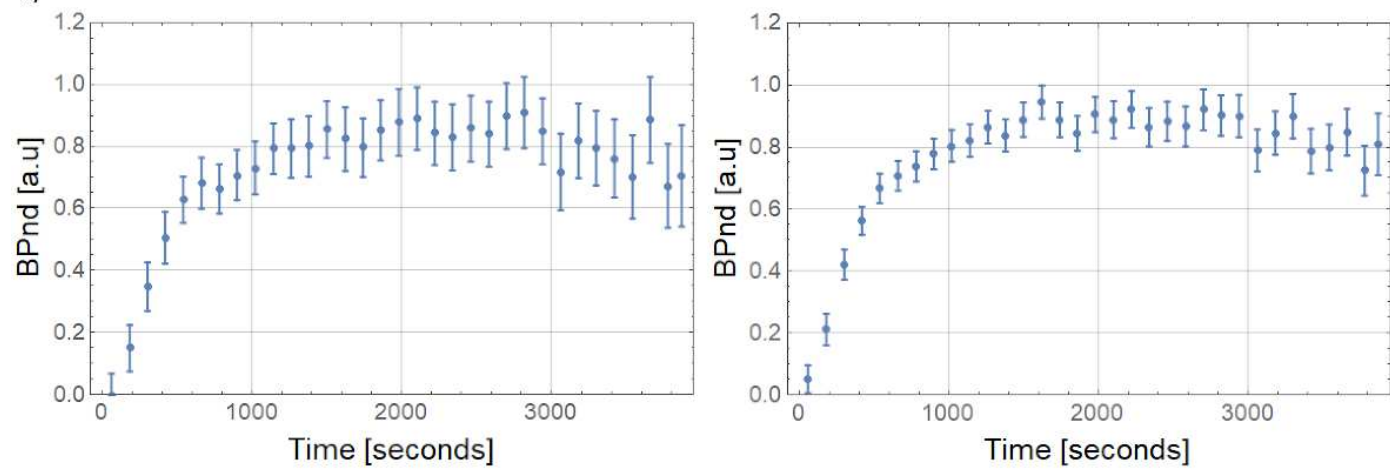

b)
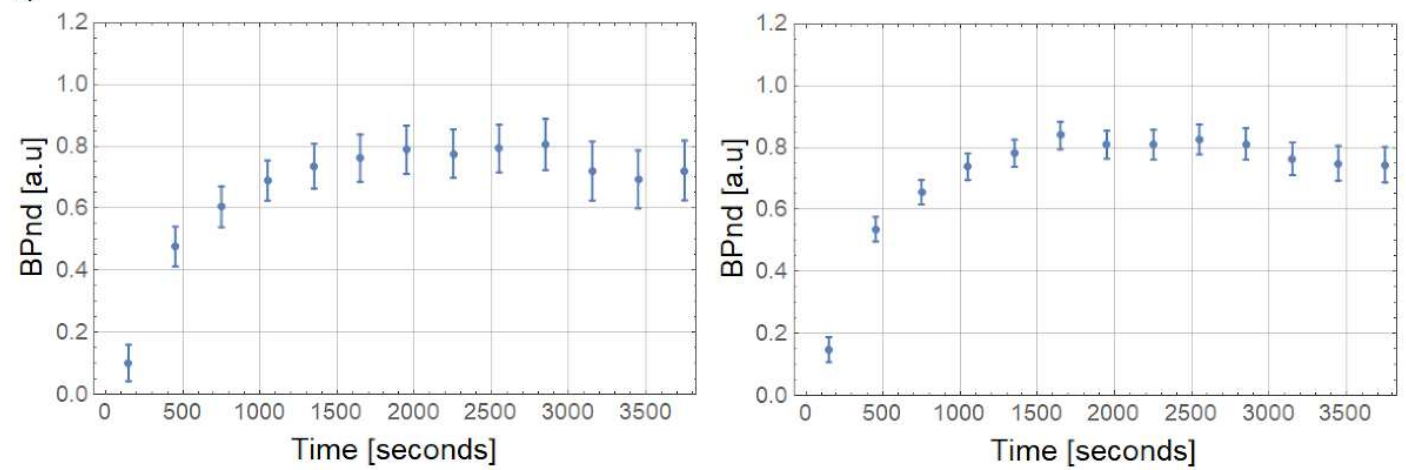

c)
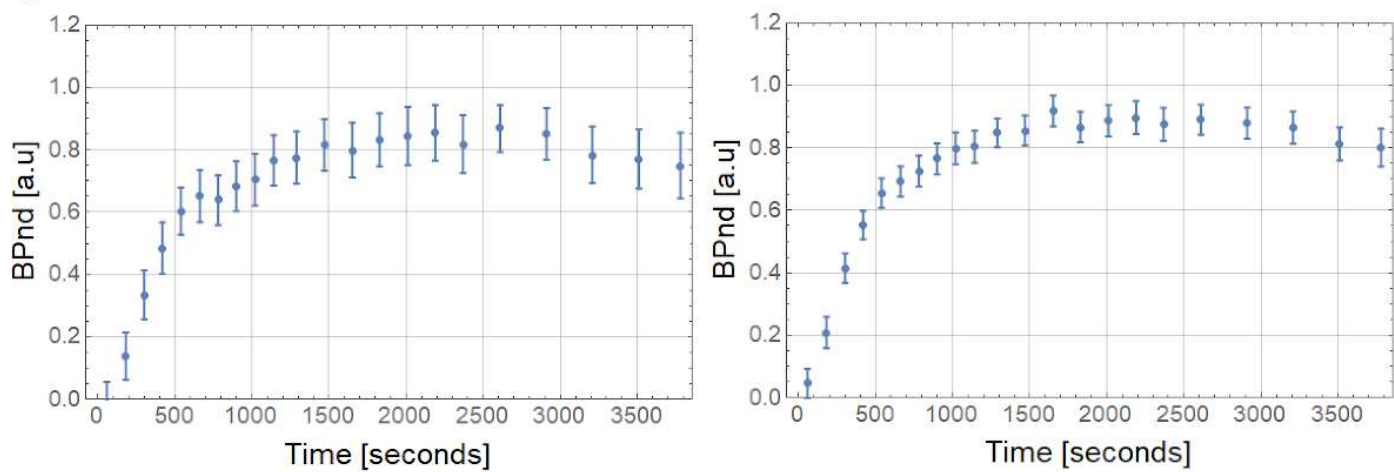

d)
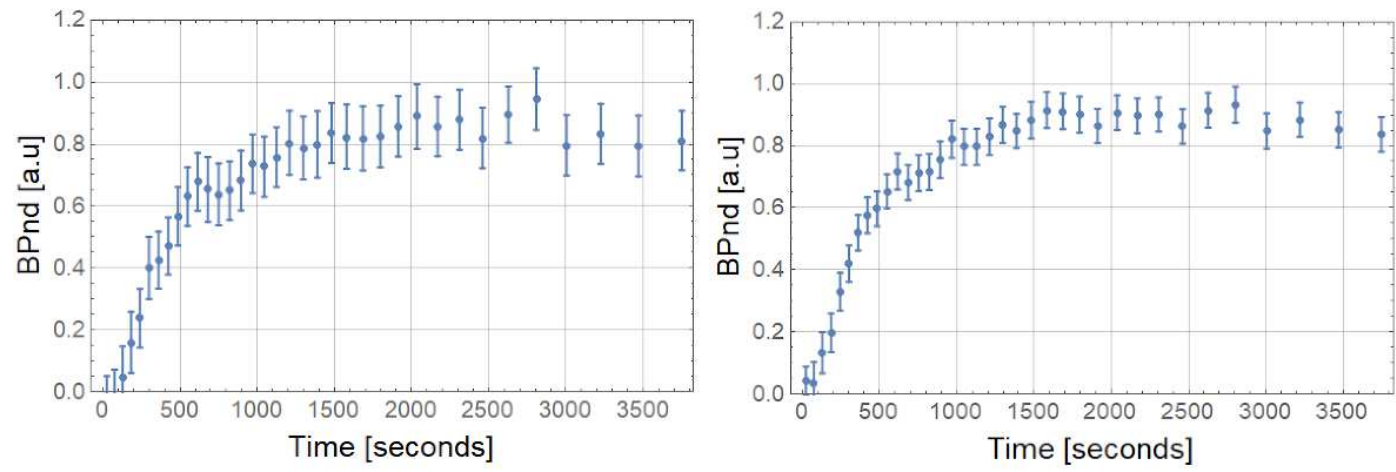

Figure 10

Values of BPND \pm SE values for ACC (left side) and Post-TI (right side) regions from a human brain study for a) Const 2 min, b) Const 5 min, c) Incr 2-3-5 min and d) Const Trues; all with PRESTO reconstruction.

\section{Supplementary Files}


This is a list of supplementary files associated with this preprint. Click to download.

- Supplement.docx 